\title{
A piecewise smooth model of evolutionary game for residential mobility and segregation
}

D. Radi ${ }^{1, a)}$ and L. Gardini', b)
${ }^{1)}$ Department of Economics and Management, University of Pisa, via Cosimo Ridolfi, 10, 56124, Pisa,
Italy
${ }^{2)}$ Department of Economics, Society, Politics, University of Urbino, via Aurelio Saffi, 2, 61029, Urbino,
Italy

(Dated: 10 April 2018)

The paper proposes an evolutionary version of a Schelling-type dynamic system to model the patterns of residential segregation when two groups of people are involved. The payoff functions of agents are the individual preferences for integration which are empirically grounded. Differently from Schelling's model, where the limited levels of tolerance are the driving force of segregation, in the current setup agents benefit from integration. Despite the differences, the evolutionary model shows a dynamics of segregation that is qualitatively similar to the one of the classical Schelling's model: segregation is always a stable equilibrium while equilibria of integration exist only for peculiar configurations of the payoff functions and their asymptotic stability is highly sensitive to parameter variations. Moreover, a rich variety of integrated dynamic behaviors can be observed. In particular, the dynamics of the evolutionary game is regulated by a one-dimensional piecewise smooth map with two kink points that is rigorously analyzed using techniques recently developed for piecewise smooth dynamical systems. The investigation reveals that when a stable internal equilibrium exists, the bimodal shape of the map leads to several different kinds of bifurcations, smooth and border collision, in a complicated interplay. Our global analysis can give intuitions to be used by a social planner to maximize integration through social policies that manipulate people's preferences for integration.

Keywords: Residential segregation; Schelling models; Piecewise-smooth map; Evolutionary games; Replicator dynamics; Bifurcations.

Residential segregation is observed in many metropolitan areas of the western society despite people express their preferences for integration. The dynamic models proposed by Schelling, see, e.g., Schelling (1969) and Schelling (1971), provide a theoretical explanation for the discrepancies between macro patterns of segregation and individual preferences for integration. Schelling's models are based on tolerances and on the threshold behavior according to which people change the residential location if the racial composition of the district cross the threshold level of tolerance and enter otherwise.

In the current paper, we propose an evolutionary version of the Schelling's neighborhood tipping model where agents decide their residential location according to their individual distributions of preferences which represent their payoff functions. Agents' location decision is modeled by a replicator dynamics according to which agents chase the best relative performance. Despite the differences with the Schelling's models, our evolutionary game shows patterns of segregation that are similar to the segregation dynamics of the Schelling's models. In fact, it shows that segregation, despite being a suboptimal equilib-

\footnotetext{
a) Electronic mail: davide.radi@unipi.it

b)Electronic mail: laura.gardini@uniurb.it
}

rium of the game, is stable and a racially diverse neighborhood is possible only for peculiar configurations of the distributions of preferences and, even when it exists, it is highly sensitive to parameter variations and initial conditions. Then, our analysis provides an evolutionary justification to the segregation dynamics observed in reality, showing that segregation can arise also through the so-called spiteful behaviors, see, e.g., Hamilton (1970), according to which segregation damages all agents but it takes place because it offers a comparative advantage to one of the two ethnic groups.

Compared to the two-dimensional dynamical Schelling's neighborhood-tipping model, see, e.g., Bischi and Merlone (2011), the evolutionary game that we propose leads to a one-dimensional map. Thus, it is easier to study, although it is a continuous and piecewise smooth map with two kink points which highly influence the observed dynamics.

We investigate the existence of the possible fixed points (equilibria of segregation and of non segregation) of the map and their local/global stability, as well as robustness both in the phase space and in the parameter space determined by two kink points of the map. We show that only one equilibrium of non segregation can be stable. However, also when all the equilibria of non segregation are repelling, interesting integrated dynamics may exist, consisting in attracting pe- 
riodic orbits or bounded aperiodic dynamics in a specified interval. We describe the relevant bifurcations depending on two kink points of the map, both smooth and non smooth, that is, transitions related to border collision bifurcations. By using the properties of the bimodal model we show relevant global bifurcations related to the fixed points, which may be also homoclinic bifurcations. Moreover, we show contact bifurcations which are related to disconnected basins of attraction, as well as to the final bifurcation (leading to no attracting integrated dynamics). Some new bifurcation structures due to the interplay between smooth bifurcations and border collision bifurcations are evidenced.

\section{INTRODUCTION}

Residential segregation between whites and blacks in the U.S. urban areas is an example of mismatch between macrobehavior and individual preferences, see, e.g., Schelling (1978). Survey data show that both whites and blacks prefer to live in integrated neighborhoods. The empirical investigations conducted in last thirty years underline that the majority of black people choose "half-black, half-white" as the favorite neighborhood racial composition, see, e.g., Clark (1991); Davis and Smith (1994); Krysan and Farley (2002). The same investigations reveal that whites have preferences for integrated neighborhoods where they are the strict majority and only a small minority of them indicate a segregated neighborhood as the favorite one, see, e.g., Clark (1991); Schuman et al. (1997). In contrast with this revealed system of individual preferences, residential segregation between blacks and whites can be observed in many U.S. metropolitan areas despite the latest, albeit mild, steps toward integration, see, e.g., Clark (1991); Massey and Denton (1993); Zhang (2004); Glaeser and Vigdor (2001); Logan, Stults, and Farley (2004).

A first explanation to this counter-intuitive phenomenon of segregation was provided by Schelling. In seminal contributions, see Schelling (1969, 1971), Schelling proposed two theoretical models that show the emergence and persistence of residential segregation in a framework in which blacks and whites have a strict preference for integration. The first model proposed by Schelling is a spacial proximity model since then known also as Schelling's checkerboard model. This microeconomic model represents one of the first examples of what nowadays are known as agent-based models, see, e.g., Epstein and Axtell (1996). According to this model, there are two types of agents, each of which does not tolerate more than a certain fraction of the other type of agents living in his/her neighborhood. Unsatisfied agents get the chance to move to a more desirable position. The process reaches its stationary state when everybody is satisfied with his/her allocation choice. Schelling's most striking finding is that moderate preferences for samecolor neighbors at the individual level can be amplified into complete residential segregation at the macro level. The reasons can be found in the logical constraints originated by individual preferences for integration. The classical example is the presence of two groups of people all agents of which want to live in a neighborhood with a strict majority of same-color neighbors. Then, residential segregation is the only configuration that satisfies both populations. This multiple-neighborhood model has been studied and developed in many ways since its introduction, see, e.g., Fagiolo, Valente, and Vriend (2007); Zhang (2011). All the follow-up contributions reveal insightful aspects of segregation, like recent studies that show the robustness of Schelling's findings by proving that residential segregation occurs even when agents have a strict preference for perfect integration, see, e.g., Pancs and Vriend (91). However, due to its complicated structure, a rigorous analysis of the model requires sophisticated mathematical tools, see Zhang (2004).

The second model proposed by Schelling is a lowdimensional one better known as neighborhood tipping model and it is purely analytical. It includes two groups of agents, one of whites and one of blacks. Agents in each group choose between residing in a (possibly) mixed neighborhood or living segregated. Each agent's preferences are used to compute threshold levels indicating the maximum tolerated number of non-co-ethnic neighbors. The distribution of tolerances differs between blacks and whites and individuals enter the district if the number of opposite-color neighbors does not exceed the tolerance level and exit otherwise. This driving force of the entry/exit dynamics is known as threshold behavior and generates a segregation pattern as the so-called neighborhood tipping process. The neighborhood tipping occurs when in an all-white neighborhood, after some blacks move in, whites start to move out because of their limited level of preference for integration and blacks, who have a higher level of tolerance, start to move in. Suddenly, the process of evolving into an all-black neighborhood begins. Similarly, an all-black neighborhood may be tipped into an all-white neighborhood and a mixedrace neighborhood can be tipped into a segregated one. The model was developed in the form of a Bayesian game with best-reply dynamics in Dokumaci and Sandholm (2006), where it takes the form of a two-dimensional dynamical system in continuous time. This setting was further developed in Bischi and Merlone (2011), where a discrete-time version, with adaptive dynamics and entry limitations, of the game in Dokumaci and Sandholm (2006) was proposed. A detailed and rigorous analysis of the latter model can be found in Radi, Gardini, and Avrutin (2014b,a), while its generalization to the case of heterogeneous distributions of tolerances is proposed in Bischi and Merlone (2011) and investigated in Radi and Gardini (2015).

The scope of the current paper is to study the evolu- 
tionary stability of the segregation patterns underlined in the Schelling's neighborhood tipping model. To this aim, an evolutionary game, in the form of a replicator dynamics (see Hofbauer and Sigmund (2003) and references therein), is proposed, where agents' payoff functions take into account the distributions of preferences empirically detected through a telephone survey and reported in Clark (1991). As in the Schelling's neighborhood tipping model, in our evolutionary game there are two populations of agents, one of whites and one of blacks, and a single neighborhood. The composition of the neighborhood evolves over time according to a replicator dynamics, with the fraction of whites that increases whenever whites' satisfaction for the current composition of the neighborhood is higher than blacks' one and decreases in the opposite case. The equilibria of the model are segregation and the ethnic composition of the neighborhood for which whites' satisfaction equals blacks' one. There are two main differences with respect to the classical tipping model by Schelling. First of all, in building this evolutionary game we model peoples' preferences for integration instead of tolerances for unlike neighbors. Secondly, agents reside in the neighborhood as long as their level of satisfaction is higher than the one of the members of the other population, despite their tolerance level may be exceeded. Then, in the classical Schelling model it is the more tolerant population that can penetrate and tip the composition of the neighborhood. Differently, in the current model people adopt a social-learning scheme, see, e.g., Bossan, Jann, and Hammerstein (2015), and to reside in a neighborhood it is not only a matter of single-agent attitudes towards integration but it is a matter of being more satisfied with the current ethnic composition of the neighborhood, than the other ones are. Therefore, in our evolutionary setup it is the population that has a higher level of satisfaction that can penetrate and tip the composition of the neighborhood.

The analysis reveals that integration is possible only for certain configurations of the individual preferences. It occurs when blacks' satisfaction for living in an integrated neighborhood where the whites are a large majority is higher than whites' one. The composition of a racially mixed neighborhood can be stable over time, it is the case of an equilibrium of integration, or not, which is the case of a periodic or aperiodic attractor of integration. Notwithstanding the existence of an equilibrium representing an integrated neighborhood, the risk of segregation always persists and the occurrence of global bifurcations can substantially reduce the set of initial conditions from which a pattern of integration originates. In fact, an all-white neighborhood represents a stable equilibrium for any configuration of the distributions of preferences consistent with the empirical observations illustrated in Clark (1991). This occurs because whites, unlike blacks, have positive preferences for living segregated. On the contrary, an all-black neighborhood is a stable equilibrium only when blacks have higher prefer- ences than whites for neighborhoods with a large majority of blacks. Another distinctive feature of our model is the equilibrium composition of the integrated district which, when it exists and is stable, includes a larger majority of whites. This seems to be consistent with the empirical data provided by the U.S. Census Bureau. ${ }^{1}$

The segregation patterns and the global dynamics of our evolutionary game are similar to the ones of the Schelling's neighborhood tipping model. As in the Schelling's model, the risk of segregation cannot be eliminated, integrated residential patterns are inherently sensitive to small variations in the distributions of preferences and in the initial composition of the neighborhood and segregation is attainable despite being highly undesirable at the individual level. In fact, the distributions of preferences of our evolutionary game underline that an equilibrium of residential integration can be at the same time the social optimum and unstable. On the contrary, an equilibrium of segregation can be at the same time the least-optimal one and stable. In this case, the segregation pattern is driven by the so-called spiteful behaviors, see, e.g., Hamilton (1970), which occurs when a player undertakes an action because it damages the others more than it damages himself. Together with the threshold behavior introduced by Schelling, this spiteful behavior, typical of the evolutionary games, provides a game-theoretic justification on individual grounds for the patterns of residential segregation that are empirically observed.

A social planner that wants to maximize integration can benefit from the global analysis of our model. The normative relevance is mainly focused on how to shape the distribution of preferences of the populations to achieve integration. Social events, education for integration and many other initiatives or social policies can change people's preferences for living in an integrated or segregated district. Our analysis provides indications of the effectiveness of these activities, how they should be tuned and their possible achievements. A further normative issue concerns how to calibrate and when to stop a reward scheme aimed to incentivize people to move to live in an integrated neighborhood.

As already remarked, the evolutionary game here proposed takes the form of a continuous and piecewise smooth one-dimensional map, with two kink points which highly influence the system dynamics. We shall perform a detailed analysis of the possible fixed points of non segregation, and the related local/global stability, showing that only one equilibrium of integration can be attracting. Moreover, when the equilibrium is repelling, interesting integrated dynamics may exist, and we describe some relevant bifurcations depending on two parameters (the two kink points of the map).

Since the map is piecewise smooth, standard smooth bifurcations may occur besides the border collision bifur-

\footnotetext{
${ }^{1}$ See, http://www.census.gov/mycd/.
} 
cations (BCBs for short henceforth) ${ }^{2}$. In particular we prove that the appearance of equilibria of non segregation can occur only via fold BCBs and only one of them can be asymptotically stable. It may lose stability through a smooth flip bifurcation (also s-flip for short) and complicated integrated dynamics can occur.

The bifurcations occurring in maps of this kind, unimodal or bimodal, are different from those occurring in smooth systems, and are characterized by an interplay between standard smooth bifurcations and border collisions (as we shall also see in our dynamic model). For example, a cycle which undergoes a border collision may lead to a cycle of the same period (in which case we call it persistent $\mathrm{BC}$ ) or to an attracting cycle of different period or directly to a chaotic attractor (in which case we have a $\mathrm{BCB}$ ), and we shall see several occurrences of this kind, investigating the dynamics associated with a two-dimensional parameter plane.

A further characteristic of piecewise smooth systems is that a chaotic attractor (which in one-dimensional continuous maps is given by an interval or $k$-cyclical intervals, see for example Avrutin, Sushko, and Gardini (2014)) may be persistent under parameter variations. Following the terminology adopted in Banerjee, Yorke, and Grebogi (1998), this phenomenon is called robust chaos and it cannot occur in smooth systems. Our system is piecewise smooth and the numerical investigation shows robustness of the complex dynamics. Also the appearance of repelling equilibria via fold BCBs may lead directly to bounded, but periodic or aperiodic, robust integrated dynamics.

Recall that the investigation of the dynamic result of a BC of a fixed point or of a $k$-cycle of a map can take advantage of the skew-tent map as a normal form. In short, the relevant information is the slope of the function that defines the dynamic model on the left and right sides of the fixed point which undergoes collision with one kink point. Similarly holds for the BC of a $k$-cycle, considering the $k$-th iterate of the map in the involved kink point (for which it corresponds to a $\mathrm{BC}$ of a fixed point). The result of a $\mathrm{BC}$ in this case can be described by using the two slopes, see Maistrenko, Maistrenko, and Vikul (1998), the survey Sushko, Avrutin, and Gardini (2016) and references therein. The skew-tent map as a normal form is also useful in a bimodal system, as shown for the bimodal piecewise linear case in Panchuk et al. (2013).

The remainder of the paper is organized as follows. Section II constructs the evolutionary model of segregation starting from empirical data. Section III presents the analytical results about the equilibria of the model with special emphasis on the role of the distributions of preferences. The system can have at most three equilibria of integration, and at most one can be attracting and may

\footnotetext{
${ }^{2}$ Following the term introduced by Nusse and Yorke, see Nusse and Yorke (1995).
}

become repelling via smooth flip bifurcation. The global scenarios that can occur are classified in four different types, for each of which we determine the characteristics. The more interesting (and difficult one) is Scenario 4 related to three equilibria of non segregation. This scenario is investigated in Section IV, where we present a global analysis of the dynamics that underlines the sensitivity of the integration patterns to both parameters and initial conditions. We investigate the local/global stability of the relevant equilibrium of non segregation, or different bounded integrated dynamics, describing the structure of the basins of attraction of the attracting sets. We show that disconnected basins of attraction appear via global bifurcations. That is, through a transition which is not related to the eigenvalues of the cycles, but to the global character of the map via the kink points and their images (also called critical points), mainly when they merge with a periodic point. Section V concludes.

\section{MODEL SETUP}

The residential mobility of whites and blacks is driven by the presence of like-color neighbors. Some white people like to live in a district where $10 \%$ of the population is black, others in a district where $20 \%$ of the population is black and so on. The same occurs for the black people. A telephone survey conducted in the USA, and reported in Clark (1991), aims to describe these preferences. The survey is based on the simple question "What mixture of people would you prefer? Would you prefer a neighborhood that is... (combinations of $100 \%$ white, 90\% white and $10 \%$ black, and so on through $100 \%$ black were read to respondents)". Answering this question a citizen of a city specifies his/her ideal composition of a neighborhood. This information is used to obtain two distributions of preferences, one for whites and one for blacks, for the ethnic composition of the neighborhood. The empirical investigation reveals that blacks and whites have heterogeneous preferences for integration.

These distributions of preferences are used as a proxy to obtain two continuous functions which depend on the fraction of blacks in the neighborhood that we denote by $x \in[0,1]$ and represent agents' satisfaction. The one of whites is a bimodal function. It has a first peak in $x$ equal to zero, which means that whites have positive preferences for living in a district populated by like-color neighbors only, a second peak in $x=d_{R}$, where $d_{R}$ is a positive value less than 0.5 , which indicates that whites like to live in a district in which they are the strict majority, it has a relative minimum point in $x=d_{L}$, with $d_{L}<d_{R}$, and it values zero in correspondence of a neighborhood populated by blacks only, i.e. whites do not like leaving in districts populated entirely by blacks. Concerning blacks, the empirical distribution of preferences for integration is a unimodal function. It has a peak in $x=d_{1}$, where $d_{1} \approx 0.5$, and it values zero in correspondence of segregated neighborhoods. This means that 
blacks like to live in an half-white, half-black district.

As the level of integration is only one of the many factors that influence the residential mobility of agents, we follow Zhang (2004) and we assume that an agent's payoff (utility) has two parts: an endogenous term, $W(\cdot)$ for whites and $B(\cdot)$ for blacks, that depends on how many like-color neighbors he/she has in the neighborhood, and an exogenous term $\delta$ that captures the value of other relevant characteristics of the neighborhood. ${ }^{3}$ The parameter $\delta$ is assumed to be independent across agents, i.e. it is the same for whites and blacks. ${ }^{4}$ Let us point out that $W(\cdot)$ and $B(\cdot)$ reflect at the same time the distributions of preferences for other-color neighbors and the weight that these distributions have on the residential choice of whites and blacks, respectively. In accordance to the empirical observations in Clark (1991), we assume that $W(\cdot)$ is a non-negative piecewise linear bimodal function of $x$ given by

$$
W(x)= \begin{cases}W_{L}(x)=a_{L} x+\mu_{L} ; & 0 \leq x \leq d_{L} \\ W_{M}(x)=a_{M} x+\mu_{M} ; & d_{L} \leq x \leq d_{R} \\ W_{R}(x)=a_{R} x+\mu_{R} ; & d_{R} \leq x \leq 1\end{cases}
$$

and

$$
\begin{gathered}
a_{L}<0, \quad 0<\mu_{L}, \quad 0<d_{L}<d_{R}<0.5, \\
a_{R}<0, \quad \mu_{R}=-a_{R}, \quad a_{L} d_{L}+\mu_{L}>0
\end{gathered}
$$

while the other two parameters of the middle branch $W_{M}(x)$ are imposed in order to ensure the continuity condition of $W(x)$ at the kink points $d_{L}$ and $d_{R}$. This leads to

$$
\begin{aligned}
a_{M} & =\frac{\mu_{R}-\mu_{L}+a_{R} d_{R}-a_{L} d_{L}}{d_{R}-d_{L}} \\
\mu_{M} & =\frac{\left(a_{R}-a_{L}\right) d_{L} d_{R}+\mu_{L} d_{R}-\mu_{R} d_{L}}{d_{R}-d_{L}}
\end{aligned}
$$

These assumptions ensure that $W(x)$ is downward sloping in $\left(0, d_{L}\right)$ and $\left(d_{R}, 1\right)$, while it is upward sloping in $\left(d_{L}, d_{R}\right), W(1)=0$ and $W(x)$ is non negative in $[0,1]$. Instead, following again the empirical evidences in Clark (1991), we assume that $B(\cdot)$ is a piecewise linear unimodal function of $x$ given by

$$
B(x)= \begin{cases}B_{L}(x)=a x ; & x \leq d_{1} \\ B_{R}(x)=\frac{a d_{1}}{1-d_{1}}(1-x) ; & x>d_{1}\end{cases}
$$

where

$$
a>0, \quad d_{1}=0.5 .
$$

\footnotetext{
${ }^{3}$ Many other neighborhood characteristics beyond race and ethnicity influence the residential choice of individuals, see, e.g., Harris (1999).

${ }^{4}$ Zhang, see Zhang (2004), indicates that the agent's payoff can be interpreted as how much he/she likes to pay for a residential location in a neighborhood.
}

These assumptions ensure that $B(x)$ is upward sloping in $(0,0.5)$, downward sloping in $(0.5,1)$, non negative in $[0,1]$ and $B(1)=B(0)=0$. The condition $d_{1}=0.5$ is justified by empirical evidences, nevertheless other values of $d_{1}$ would not change the results that follow as long as the condition $d_{L}<d_{R}<d_{1}$ is satisfied.

Following an evolutionary approach and considering a single neighborhood, at each interval of time the number of whites that choose to leave in the neighborhood is proportional to the previous-period payoff of those whites who lived there. The same occurs for the blacks. Then, the fraction of blacks in the neighborhood is updated accordingly. In particular, considering a neighborhood with a fraction of blacks at time $t \in \mathbb{N}$ equal to $x_{t}$, we assume that $x_{t}$ evolves over time according to the following one-dimensional piecewise smooth map:

$$
\begin{aligned}
x_{t+1} & =T\left(x_{t}\right), \quad \text { where } \\
T\left(x_{t}\right) & :=x_{t} \frac{B\left(x_{t}\right)+\delta}{B\left(x_{t}\right) x_{t}+\left(1-x_{t}\right) W\left(x_{t}\right)+\delta}
\end{aligned}
$$

The dynamic equation (6) is a so-called replicator dynamics (commonly adopted in evolutionary game theory and population dynamics to describe the Darwinian selection of species and in economics as a strategy selection mechanism, see, e.g., Hofbauer and Sigmund (2003) and reference therein) according to which the fraction of blacks in the neighborhood increases as long as their payoff (satisfaction with the current composition of the neighborhood), which is given by $B\left(x_{t}\right)+\delta$, is higher than the payoff of the whites, which is given by $W\left(x_{t}\right)+\delta$, and decreases in the opposite case. Then, the model describes the dynamics of integration/segregation in an evolutionary fashion with blacks and whites who have heterogeneous preferences for integration. The role played by these preferences can differ between blacks and whites and it is possible to represent a different degree of importance of the ratio of color-like neighbors in the decision process between the two groups of people by shaping the two payoff functions in different ways, i.e. by tuning the parameters of the function $B(x)$ and $W(x)$ in such a way that one is much larger than the other in relation to $\delta$ (without loss of generality, in all the figures of the paper we have used $\delta=0.1$ ).

In Figure 1 and Figure 2 we show some of the possible shapes of functions $W(x), B(x)$ and $T(x)$ to be commented in the following.

\section{PROPERTIES OF THE MAP}

Map $T$ in (6) is to be considered in $I=[0,1]$. Values of $x$ out of such interval are not of interest for the considered application. Let us point out that $B(x) \geq 0$ and $W(x) \geq$ $0 \forall x \in[0,1]$. It follows that map $T$ is invariant in $I$ : $T(I)=I$. Then, we are interested in the fixed points of $T(x)$ inside $I$. To this goal, we focus on the subspace $\left(0, d_{1}\right)$ of $I$, in $\left(d_{1}, 1\right)$ there are not isolated fixed points as we will see later, and we shall denote as left, middle 

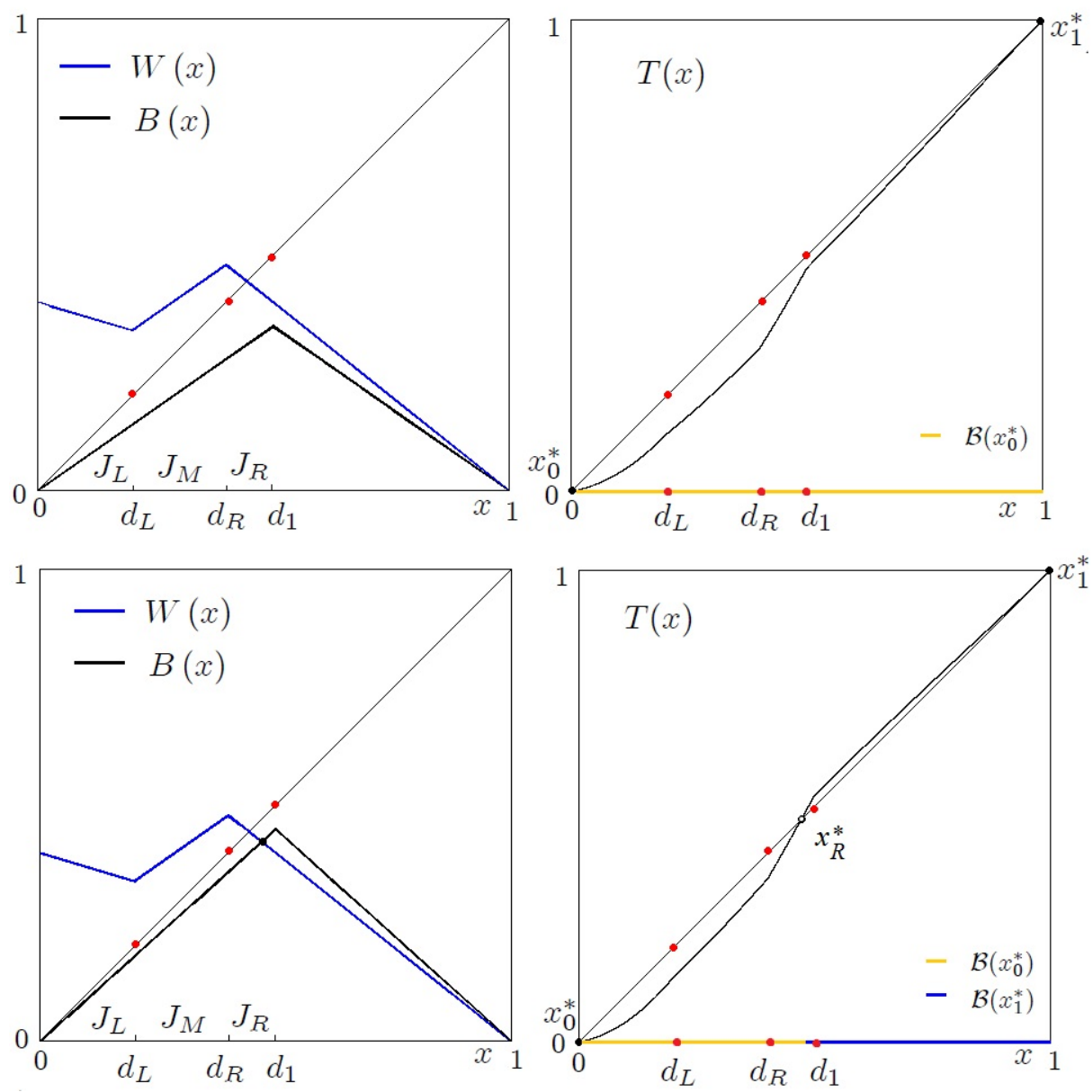

FIG. 1. In the left column the two payoff functions: $B(x)$ in black and $W(x)$ in blue. In the right column the graph of map $T$. In both columns red dots indicate the kink points $d_{L}, d_{R}$ and $d_{1}$. The black dots are the attracting fixed points and the empty dots are the repelling fixed points. The blue interval is the basin of attraction of the equilibrium of segregation $x_{1}^{*}$, the orange interval is the basin of attraction of the equilibrium of segregation $x_{0}^{*}$. First row, Scenario 1 in Theorem 2 , parameters: $a=0.7, a_{L}=-0.3, a_{R}=-0.8, \mu_{L}=0.4, d_{L}=0.2, d_{R}=0.4$. Second row, Scenario 2 in Theorem 2, parameters: $a=0.9$, $a_{L}=-0.3, a_{R}=-0.8, \mu_{L}=0.4, d_{L}=0.2, d_{R}=0.4$.

and right branch of map $T$ those defined in the intervals $J_{L}=\left(0, d_{L}\right), J_{M}=\left(d_{L}, d_{R}\right)$ and $J_{R}=\left(d_{R}, d_{1}\right)$, in which the piecewise smooth map $T$ is smooth. That is, the intervals separated by the kink points of $T$, as they will turn to be the relevant ones in describing the dynamics of the map.

From the definition of map $T$ it is immediate to see that $x_{0}^{*}=0$ and $x_{1}^{*}=1$ are fixed points (since by construction $T(0)=0$ and $T(1)=1)$, and clearly represent equilibria $^{5}$ of segregation (all whites in $x_{0}^{*}$ and all blacks in $x_{1}^{*}$ ). While an internal fixed point $x^{*}$ satisfies the equality $B\left(x^{*}\right)=W\left(x^{*}\right)$ and represents an equilibrium of non segregation (or equivalently an equilibrium of integration). ${ }^{6}$ It results that according to the shape of the

\footnotetext{
${ }^{5}$ In this paper, we use equilibrium as synonymous of fixed point.

6 Thus the intersection points of the two functions $B(x)$ and $W(x)$ in $(0,1)$ represent equilibria of non segregation.
}

distribution of preferences of the two populations, we can or we cannot have fixed points in $(0,1)$. And in any case they may be at most three, more properly, at most one in each of the intervals separated by the kink points of the map, as it is specified in the following Theorem.

Theorem 1 Given map $T$ as defined in (6) and the parameter conditions (2), (3) and (5), $x_{0}^{*}=0$ and $x_{1}^{*}=1$ are always fixed points of the map. Moreover,

1. Each open interval in which map $T$ is smooth, either is filled with fixed points or at most one fixed point of non segregation can exist. In particular, the points

$$
x_{\xi}^{*}=\frac{\mu_{\xi}}{a-a_{\xi}}, \text { for } \xi \in\{L, M, R\}
$$

are fixed points of non segregation when $x_{\xi}^{*} \in J_{\xi}$.

2. If $B\left(d_{1}\right)>W\left(d_{1}\right)$, then at least one fixed point of $T$ in $(0,1)$ exists. Specifically: 

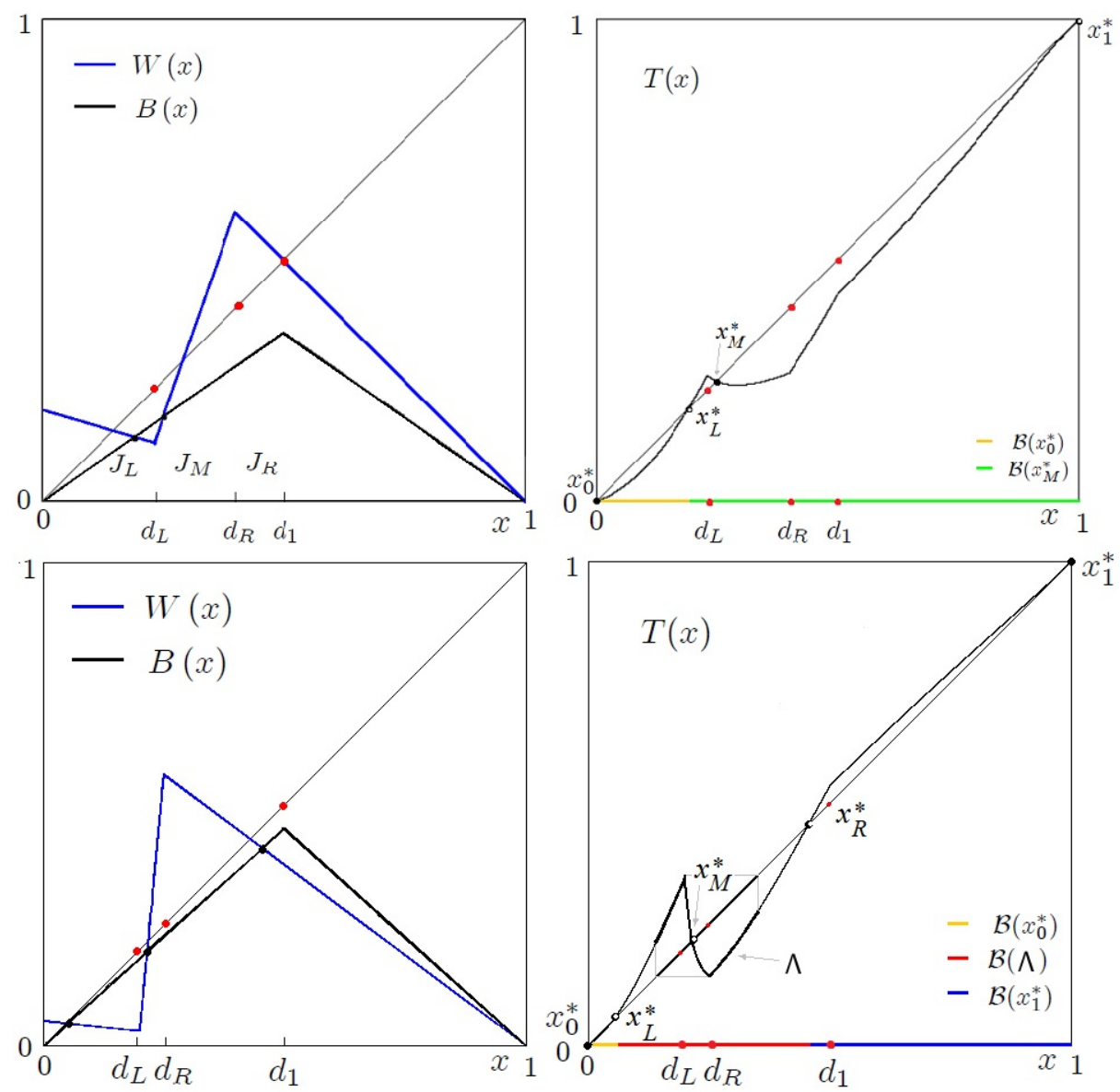

FIG. 2. The notation is as in Figure 1. First row, Scenario 3 in Theorem 2, parameters: $a=0.7, a_{L}=-0.3, a_{R}=-1$, $\mu_{L}=0.19, d_{L}=0.23, d_{R}=0.4$, the green interval is the basin of attraction of the equilibrium of integration $x_{M}^{*}$. Second row, Scenario 4 in Theorem 2, parameters: $a=0.9, a_{L}=-0.1, a_{R}=-0.75, \mu_{L}=0.05, d_{L}=0.2, d_{R}=0.25$, the red interval is the basin of attraction of the inner attractor, here a chaotic interval.

(2a) If $W\left(d_{L}\right)>B\left(d_{L}\right)$ and $W\left(d_{R}\right)>B\left(d_{R}\right)$, then $x_{R}^{*}=\frac{\mu_{R}}{a-a_{R}} \in\left(d_{R}, d_{1}\right)$ is the only fixed point of non segregation;

(2b) If $W\left(d_{L}\right)>B\left(d_{L}\right)$ and $W\left(d_{R}\right)<B\left(d_{R}\right)$, then $x_{M}^{*}=\frac{\mu_{M}}{a-a_{M}} \in\left(d_{L}, d_{R}\right)$ is the only fixed point of non segregation;

(2c) If $W\left(d_{L}\right)<B\left(d_{L}\right)$ and $W\left(d_{R}\right)>B\left(d_{R}\right)$, then the three fixed points of non segregation in (7) exist;

(2d) If $W\left(d_{L}\right)<B\left(d_{L}\right)$ and $W\left(d_{R}\right)<B\left(d_{R}\right)$, then $x_{L}^{*}=\frac{\mu_{L}}{a-a_{L}} \in\left(0, d_{L}\right)$ is the only fixed point of non segregation.

3. If $W\left(d_{1}\right)>B\left(d_{1}\right)$, then the map has either no fixed points of non segregation or two fixed points of non segregation. Specifically:

(3a) If $B\left(d_{L}\right)<W\left(d_{L}\right)$, then either no internal fixed point exists or $\left[d_{1}, 1\right]$ is filled with fixed points;

(3b) If $B\left(d_{L}\right)>W\left(d_{L}\right)$, then map $T$ has two fixed points of non segregation given by $x_{L}^{*}=$

$$
\frac{\mu_{L}}{a-a_{L}} \in\left(0, d_{L}\right) \text { and } x_{M}^{*}=\frac{\mu_{M}}{a-a_{M}} \in\left(d_{L}, d_{R}\right) .
$$

Proof. By construction $x_{0}^{*}=0$ and $x_{1}^{*}=1$ are two fixed points of $T$, while for the internal fixed points let us point out that $T\left(x^{*}\right)=x^{*}$, with $x^{*} \in(0,1)$, implies $W\left(x^{*}\right)=B\left(x^{*}\right)$. From the linearity of $B(x)$ and $W(x)$ in the intervals $J_{\xi}$ for $\xi \in\{L, M, R\}$ and $\left(d_{1}, 1\right)$, it follows that in each of them the map $T$ has either one fixed point, or no fixed point, or the fixed points fill the interval. Solving for $B(x)=W(x)$ the first point of the Theorem follows and in particular we have:

-: In $J_{L}=\left(0, d_{L}\right), x_{L}^{*}=\frac{\mu_{L}}{a-a_{L}} \geq 0\left(\right.$ since $\left.a_{L}<0<a\right)$ is a fixed point of map $T$ if and only if $x_{L}^{*} \in\left[0, d_{L}\right]$ (square brackets are used thanks to the continuity of $B(x)$ and $W(x)$ in $[0,1]$ ), from which we obtain the existence condition $\mu_{L} \leq\left(a-a_{L}\right) d_{L}$ (or equivalently $\left.B\left(d_{L}\right) \geq W\left(d_{L}\right)\right)$;

-: In $J_{M}=\left(d_{L}, d_{R}\right)$, if $a_{M} \neq a$ then $x_{M}^{*}=\frac{\mu_{M}}{a-a_{M}}$ is a fixed point of map $T$ if and only if $x_{M}^{*} \in$ $\left[d_{L}, d_{R}\right]$, from which we obtain the existence condition $d_{L}<\frac{\mu_{M}}{a-a_{M}}<d_{R}$. The inequalities are sat- 
isfied in only two cases: either $a>a_{M}, \mu_{M}>0$ and $0<\left(a-a_{M}\right) d_{L}<\mu_{M}<d_{R}\left(a-a_{M}\right)$ [equivalently $0<B\left(d_{L}\right)<W\left(d_{L}\right)<W\left(d_{R}\right)<B\left(d_{R}\right)$ ], or $a<a_{M}, \mu_{M}<0$ and $0>d_{L}\left(a-a_{M}\right)>\mu_{M}>$ $d_{R}\left(a-a_{M}\right)$ [equivalently $W\left(d_{R}\right)>B\left(d_{R}\right)>$ $\left.B\left(d_{L}\right)>W\left(d_{L}\right)>0\right]$. Moreover, for $\mu_{M}=0$ and $a=a_{M}$, we have $B(x)=W(x) \forall x \in\left[d_{L}, d_{R}\right]$, then the fixed points of $T$ fill the segment $\left[d_{L}, d_{R}\right]$;

-: In $J_{R}=\left(d_{R}, d_{1}\right), x_{R}^{*}=\frac{\mu_{R}}{a-a_{R}}$ is a fixed point of map $T$ if and only if $x_{R}^{*} \in\left[d_{R}, d_{1}\right]$, from which we obtain the existence conditions $d_{R}<\frac{\mu_{R}}{a-a_{R}}<d_{1}$, which, since $\mu_{R}>0$ and $a_{R}<0<a$, are satisfied in only one case: $\left(a-a_{R}\right) d_{R}<\mu_{R}<d_{1}\left(a-a_{R}\right)$ (or equivalently $B\left(d_{R}\right)<W\left(d_{R}\right)$ and $W\left(d_{1}\right)<$ $\left.B\left(d_{1}\right)\right)$;

-: In $\left[d_{1}, 1\right]$, since $B(1)=W(1)$, by linearity of $B(x)$ and $W(x)$ in this interval we have that there are no other intersection points (as it must hold either $B(x)<W(x)$ or $\left.B(x)>W(x) \forall x \in\left[d_{1}, 1\right)\right)$, or $B(x)=W(x) \forall x \in\left[d_{1}, 1\right]$, i.e. a continuum of fixed points fills the interval.

Since $W\left(d_{1}\right)>B\left(d_{1}\right)$ implies $W\left(d_{R}\right)>B\left(d_{R}\right)$, points 2 and 3 of the Theorem follows.

Thus, the fixed points of non segregation, when existing and in finite number, can be three, two or one. Moreover, they may represent stable configurations of the population of the district where the number of blacks is always less than half of the total population of the district. Then, although blacks and whites can cohabit in a district, we expect that the configuration of the population is such that the whites are the majority and the blacks are the minority. The alternative is either segregation, a district populated by either all whites or all blacks, or bounded dynamics (such as periodic or aperiodic orbit) internal to $(0,1)$. In the latter case, despite the absence of stable equilibria of non segregation, some level of integration is achieved. However, it is the local (and global) stability properties of these internal fixed points and internal attractors that indicate the real possibility of integration between the two populations. The following Theorem provides a general statement that describes some possible scenarios of integration/segregation and indicates under which condition an equilibrium is locally stable.

Theorem 2 Given map $T$ as defined in (6) and the parameter conditions (2),(3) and (5), the fixed point of segregation $x_{0}^{*}=0$ is always locally stable and the following scenarios may occur.

- Scenario 1: If there are no internal fixed points, then $x_{0}^{*}$ is globally attracting and $x_{1}^{*}$ is repelling;

- Scenario 2: If there is a unique internal fixed point of non segregation $x_{\xi}^{*}=\frac{\mu_{\xi}}{a-a_{\xi}} \in J_{\xi}$, for $\xi \in\{L, M, R\}$, then it is necessarily repelling and it separates the basins of attraction of the locally attracting fixed points of segregation $x_{0}^{*}$ and $x_{1}^{*}$;
- Scenario 3: If there are two internal fixed points of non segregation, i.e. $x_{L}^{*}$ and $x_{M}^{*}$, then $x_{L}^{*}$ is repelling, the fixed point of segregation $x_{0}^{*}$ is locally attracting, the second fixed point of segregation $x_{1}^{*}$ is repelling, while $x_{M}^{*}$ is locally stable for $T^{\prime}\left(x_{M}^{*}\right) \geq-1$ and it may lose stability via a smooth flip bifurcation (occurring when $T^{\prime}\left(x_{M}^{*}\right)=-1$ );

- Scenario 4: If there are three internal fixed points of non segregation then $x_{L}^{*}$ and $x_{R}^{*}$ are repelling, the fixed points of segregation $x_{0}^{*}$ and $x_{1}^{*}$ are locally attracting, while $x_{M}^{*}$ is locally attracting for $T^{\prime}\left(x_{M}^{*}\right) \geq-1$ and it may lose stability via a smooth flip bifurcation (occurring when $T^{\prime}\left(x_{M}^{*}\right)=-1$ ).

Proof. By straightforward calculations we obtain

$$
T^{\prime}\left(x_{0}^{*}\right)=\frac{\delta}{\mu_{L}+\delta} \in(0,1)
$$

so that $x_{0}^{*}$ is always locally attracting. Moreover, from Theorem 1 it follows that the number of internal fixed points can be zero, as it is assumed in Scenario 1, one, as it is assumed in Scenario 2, two, as it is assumed in Scenario 3, and three, as it is assumed in Scenario 4. Then, let us prove each single scenario.

For Scenario 1: let us consider the case of no internal fixed points, which implies $B(x)<W(x)$ for all $x \in$ $(0,1)$. It follows that $T(x)<x$ for all $x \in(0,1)$. Since $T$ is invariant in $I=[0,1]$, then $x_{0}^{*}$ is globally attracting, i.e. it attracts all the points in $I \backslash x_{1}^{*}$ and $x_{1}^{*}$ is repelling.

For Scenario 2: let $x_{\xi}^{*} \in(0,1)$ be the unique internal fixed point of map $T(x)$, given by $x_{\xi}^{*}=\frac{\mu_{\xi}}{a-a_{\xi}} \in J_{\xi}$, for $\xi \in\{L, M, R\}$. Then it must be $B(x)<W(x)$ for all $x \in\left(0, x_{\xi}^{*}\right)$ and $B(x)>W(x)$ for all $x \in\left(x_{\xi}^{*}, 1\right)$, which implies that $x_{\xi}^{*}$ is a repelling fixed point, $\mathcal{B}\left(x_{0}^{*}\right)=\left[0, x_{\xi}^{*}\right)$ is the basin of attraction of $x_{0}^{*}$, and $\mathcal{B}\left(x_{1}^{*}\right)=\left(x_{\xi}^{*}, 1\right]$ is the basin of attraction of $x_{1}^{*}$.

Before looking at Scenarios 3 and 4, let us consider the stability properties of the three internal equilibria $x_{\xi}^{*}$, with $\xi \in\{L, M, R\}$. By straightforward calculations we obtain (note that at any fixed point $x_{\xi}^{*} \in J_{\xi}$ for $\xi \in$ $\{L, M, R\}$ it is $\left.B\left(x_{\xi}^{*}\right)=W\left(x_{\xi}^{*}\right)\right)$ :

$$
\begin{aligned}
T^{\prime}\left(x_{\xi}^{*}\right) & =1+x_{\xi}^{*}\left(1-x_{\xi}^{*}\right)\left[\frac{B^{\prime}\left(x_{\xi}^{*}\right)-W^{\prime}\left(x_{\xi}^{*}\right)}{B\left(x_{\xi}^{*}\right)+\delta}\right] \\
& =1+x_{\xi}^{*}\left(1-x_{\xi}^{*}\right) \frac{a-a_{\xi}}{a x_{\xi}^{*}+\delta}, \quad \forall \xi \in\{L, M, R\}(9)
\end{aligned}
$$

so that $T^{\prime}\left(x_{L}^{*}\right)>1$ and $T^{\prime}\left(x_{R}^{*}\right)>1$ always hold, i.e. both $x_{L}^{*}$ and $x_{R}^{*}$ are repelling, when existing. Differently, when $W\left(x_{M}^{*}\right)=B\left(x_{M}^{*}\right)$ holds it must be $a<a_{M}$, and thus it is always $T^{\prime}\left(x_{M}^{*}\right)<1$, so that the fixed point $x_{M}^{*}$ may be attracting (if $T^{\prime}\left(x_{M}^{*}\right)>-1$ ) or repelling (when $T^{\prime}\left(x_{M}^{*}\right)<-1$ ). The stability condition for $x_{M}^{*}$, 
$T^{\prime}\left(x_{M}^{*}\right)>-1$, holds for

$$
\frac{\left(a-a_{M}-\mu_{M}\right) \mu_{M}}{a \mu_{M}+\delta\left(a-a_{M}\right)}>-2
$$

and clearly we have the condition at which a smooth flip bifurcation of $x_{M}^{*}$ may occur $\left(T^{\prime}\left(x_{M}^{*}\right)=-1\right)$, given by

$$
\frac{\left(a-a_{M}-\mu_{M}\right) \mu_{M}}{a \mu_{M}+\delta\left(a-a_{M}\right)}=-2 .
$$

Then let us discuss Scenarios 3 and 4. For Scenario 3: let $x_{L}^{*}$ and $x_{M}^{*}$ be the only internal fixed points, which occurs for $W\left(d_{1}\right)>B\left(d_{1}\right)$ (from Theorem 1 ). Then, by assumption no other fixed points in $\left(x_{M}^{*}, 1\right)$ can exist and by continuity of $W(x)$ and $B(x)$ in $I$, it follows that $W(x)>B(x)$ for all $x \in\left(x_{M}^{*}, 1\right)$, i.e. $T(x)<x$, which implies that $x_{1}^{*}$ is repelling. The stability properties of the other fixed points are already proved.

For Scenario 4: let us consider three internal fixed points, which holds for $B\left(d_{1}\right)>W\left(d_{1}\right)$ (from Theorem 1). Then, since $B(1)=W(1)$ and by linearity of $B(x)$ and $W(x)$ in $\left(d_{1}, 1\right)$, we have $B(x)>W(x)$ for all $x \in\left(d_{L}, 1\right)$, i.e. $T(x)>x$. Since $T$ is invariant in $I=[0,1]$, the local stability of $x_{1}^{*}$ follows. The stability properties of the other fixed points are already proved.

Several examples of the dynamic scenarios listed in Theorem 2 are shown in Figure 1 and Figure 2, as commented below. This Theorem underlines that there is only one equilibrium of non segregation, i.e. $x_{M}^{*} \in$ $\left(d_{L}, d_{R}\right)$, which can be locally stable while the equilibrium of segregation $x_{0}^{*}$ is always locally stable, which means that the risk of segregation cannot be eliminated. Such equilibrium of segregation can even be globally stable, which occurs (in Scenario 1) when there is an unbalance distribution of preferences for integration between blacks and whites, with whites that have a higher propensity for integration than blacks, i.e. $W(x)>B(x)$ $\forall x \in(0,1)$, see e.g. Figure 1(first row). In this case the willingness for integration of the whites is much higher than the one of the blacks. This gap causes an incompatibility of preferences between blacks and whites making segregation the only possible outcome. Similar to what observed by Shelling, see Schelling (1969) and Schelling (1971), segregation occurs despite being a suboptimal configuration of the population of the district, i.e. for both blacks and whites the degree of satisfaction coming from integration is higher than the one coming from segregation. For example, according to Theorem 2, segregation may occur for distributions of preferences such that $W\left(d_{R}\right)>W(0)$ and $B\left(d_{R}\right)>B(0)$, i.e. when both whites and blacks are better off in an integrated district.

This segregation dynamics, that sounds counterintuitive, can take the form of what in evolutionary game theory is called spiteful behavior, see, e.g., Schaffer (1989): the fraction of whites increases and the neighborhood is tipped into a segregated one just because this trend damages the blacks more than the whites themselves. In the end, it does not matter how large is the level of satisfaction for integration of the two groups of people, as long as whites have relative advantage over blacks in terms of preferences for integration, the number of whites in the district will increase and the number of blacks will decrease. This evolutionary dynamics of segregation indicates that integration policies aimed at increasing the degree of satisfaction of only one group of people may not produce the desired effect. More effective are integration policies that aim to standardize the desire of integration between the two populations.

Starting a comprehensive analysis of the possible patterns of segregation, let us underline that the globally stable equilibrium of segregation represents the first of the four possible scenarios underlined in Theorem 2. The second one occurs when $W(x)>B(x)$ for all $x \in\left(0, x_{\xi}^{*}\right)$ and $W(x)<B(x)$ for all $x \in\left(x_{\xi}^{*}, 1\right)$, where $x_{\xi}^{*}$, for $\xi \in\{L, M, R\}$, is the unique fixed point of non segregation, see an example in Figure 1(second row). In terms of preferences for integration, the second scenario describes a situation in which a district populated mainly by whites is preferred by whites more than by blacks. On the other way, a district populated by blacks is preferred by blacks more than by whites. Theorem 2 specifies that this configuration of preferences can only lead to segregation. Depending on the initial configuration of the population, segregation where either whites or blacks live in the district is the final outcome of the residential patterns.

So far, we have underlined that the individual preferences for integration are the main driving forces of segregation. However, there are configurations of preferences, consistent with the empirical observations in Clark (1991), that can admit forms of integration. It is the case of Scenario 3 described in Theorem 2, where whites have preferences higher than blacks for a district populated in large majority by blacks, $W(x)>B(x)$ for all $x \in\left(x_{M}^{*}, 1\right)$, while blacks prefer a district populated in a certain measure by a mixture of the two populations more than whites, $W(x)<B(x)$ for all $x \in\left(x_{L}^{*}, x_{M}^{*}\right)$. This configuration of preferences allows to have integration. Nevertheless, the empirically-observed positive preferences of whites for living in a district populated by color-like neighbors only and the zero preference that blacks have for such a configuration, makes sure that a district populated by whites is always a possibility (recall that $x_{0}^{*}$ is always locally attracting) and the risk of segregation cannot be eliminated, see e.g. Figure 2(first row).

The fourth scenario in Theorem 2 is the more complicated and uncertain one. In this case, blacks have a preference higher than whites for districts populated mainly by blacks, while whites have a higher preference than blacks for district mainly populated by whites. This configuration guarantees that segregation, either all whites or all blacks, can occur depending on the initial conditions. Nevertheless, blacks have preferences higher than whites for districts with a certain mixture of the two populations, say $B(x)>W(x)$ for all $x \in\left(x_{L}^{*}, x_{M}^{*}\right)$, and the 
situation is reversed when the percentage of blacks grows, say $W(x)>B(x)$ for all $x \in\left(x_{M}^{*}, x_{R}^{*}\right)$. Depending on the initial state, this configuration of preferences can allow to have an integration pattern. The attracting set can take the form of a fixed point of non segregation, or of periodic orbits (i.e. $k$-cycles with $k>1$ ), or of a set with complex behaviors. See e.g. Figure 2(second row) and Figure 3(b). Let us point out that periodic orbits as well as chaotic ones mean that in the long run the composition of the neighborhood is always changing, with blacks as well as whites that continuously enter and exit the neighborhood. Moving in and out is a costly activity and it requires time, however this is not an issue as the unit of time can be defined in accordance with the social changes described by the model.

Theorem 2 provides useful insights of how different configurations of the individual preferences for integration are responsible for the emergence of residential patterns of integration/segregation. The information included in this Theorem can be used to generate guidelines indicating for which configurations of the distributions of preferences, i.e. the third and the fourth scenarios, integration can occur. Nevertheless, in the third and fourth scenarios the integration can be threatened by the configuration of the basins of attraction, as well as by the regions in the parameter space related to stability of the non segregation fixed point $x_{M}^{*}$ and local or global bifurcations that make integration very sensitive to variations in the initial conditions and in the parameter values of the model. Moreover, map $T$ can generate complicated dynamics and unexpected results, which are however related to integration. In the following a global analysis of the dynamics allows us to assess the fragility of the residential patterns of integration and a comparison with the segregation dynamics of the Schelling's model underlines the robustness of our results.

\section{THE FRAGILE PATTERNS OF INTEGRATION: BIFURCATION STRUCTURE AND GLOBAL DYNAMICS OF THE MODEL}

The two Theorems of the previous section determine the conditions for the existence and local stability of the equilibria of map $T$, but it is clearly of interest to investigate the dynamic behaviors and local as well as global bifurcations that may occur, as some parameters of the model are varied. Before analyzing the possible paths and bifurcation diagrams let us describe the bifurcations that regulate the transitions between the four scenarios indicated in Theorem 2.

As we have seen from Theorem 1, a fixed point may belong to any one of the intervals $J_{L}=\left(0, d_{L}\right), J_{M}=$ $\left(d_{L}, d_{R}\right)$ and $J_{R}=\left(d_{R}, d_{1}\right)$. The endpoints of the intervals are the kink points and thus are considered as special cases. That is, whenever a kink point is also fixed, respectively periodic of any period, then a border collision is occurring to the fixed point, respectively to the cycle.
First notice that from Scenario 1 without internal equilibria the appearance of the repelling fixed point $x_{R}^{*}$ can occur only via a degenerate bifurcation of $x_{1}^{*}$. That is, a transition from repelling to attracting via a segment of fixed points (case 3(a) in Theorem 1), occurring when $W\left(d_{1}\right)=B\left(d_{1}\right)$, that is, for

$$
a_{L}=-\frac{a d_{1}}{1-d_{1}}
$$

which also implies $W(x)=B(x)$ for any $x \in\left[d_{1}, 1\right]$.

While from point 1 in Theorem 1 it follows that the appearance/disappearance of a pair of fixed points $\left(x_{L}^{*}\right.$ and $x_{M}^{*}$ or $x_{M}^{*}$ and $x_{R}^{*}$ ) cannot occur via a smooth fold bifurcation (since it would lead to a pair of equilibria in one interval $J_{\xi}$, for some $\xi$, which cannot occur), but only via a fold $\mathrm{BCB}$ involving either the kink point $d_{L}$, occurring when $W\left(d_{L}\right)=B\left(d_{L}\right)$ and related to the pair of fixed points $x_{L}^{*}$ and $x_{M}^{*}$, or involving the kink point $d_{R}$, occurring when $W\left(d_{R}\right)=B\left(d_{R}\right)$ and related to the pair of fixed points $x_{M}^{*}$ and $x_{R}^{*}$. Summarizing, a fold $\mathrm{BCB}$ can occur in two cases:

(i) Via a contact in $d_{L}$ when $W\left(d_{L}\right)=B\left(d_{L}\right)$, at which $T\left(d_{L}\right)=d_{L}$ holds (i.e. $\left.d_{L}\left(a-a_{L}\right)=\mu_{L}\right)$, leading to the condition of fold $\mathrm{BCB}$

$$
F-B C B_{L}: \quad d_{L}=d_{F B-L}:=\frac{\mu_{L}}{a-a_{L}}
$$

at which the two equilibria of integration $x_{L}^{*}$ and $x_{M}^{*}$ merge with $d_{L}$ and appear/disappear;

(ii) Via a contact in $d_{R}$ when $W\left(d_{R}\right)=B\left(d_{R}\right)$, at which $T\left(d_{R}\right)=d_{R}$ holds (i.e. $\left.d_{R}\left(a-a_{R}\right)=-a_{R}\right)$, leading to the condition of fold $\mathrm{BCB}$

$$
F-B C B_{R}: \quad d_{R}=d_{F B-R}:=\frac{-a_{R}}{a-a_{R}}
$$

at which the two equilibria of integration $x_{M}^{*}$ and $x_{R}^{*}$ merge with $d_{R}$ and appear/disappear.

As an example, we can see in Figure 3(a) a unique internal fixed point $x_{L}^{*} \in J_{L}$ which is repelling (from Scenario 2 in Theorem 2). Here, reducing the parameter $d_{R}$ a fold BCB occurs in the kink point $d_{R}$, after which a pair of new internal fixed points appear on the opposite sides of $d_{R}$, see Figure 3(b).

The bifurcation analysis so far conducted underlines that when there is a unique equilibrium of non segregation (necessarily repelling, see again Scenario 2 in Theorem 2), then a new pair of internal fixed points can occur only via a fold BCB. As recalled in the Introduction, it is also possible to detect the local stability/instability of the fixed points related to a fold BCB. In fact, by using the right and left slopes of the function $T(x)$ at the kink point involved in the fold $\mathrm{BCB}$, it is possible to determine whether a fold $\mathrm{BCB}$ leads to a pair of unstable internal fixed points or to an attracting 
$x_{M}^{*}$ (as the other equilibrium of integration is necessarily repelling, see Theorem 2). In particular, in case (i) where $W\left(d_{L}\right)=B\left(d_{L}\right)$ and the border collision condition $d_{L}\left(a-a_{L}\right)=\mu_{L}$ holds, the right derivative of the function $T(x)$ in $d_{L}$ is

$$
\begin{array}{r}
T_{+}^{\prime}\left(d_{L}\right)=1+d_{L}\left(1-d_{L}\right) \frac{a-a_{M}}{a d_{L}+\delta}, \quad \text { where } \\
a_{M}=\frac{-a_{R}\left(1-d_{R}\right)-a d_{L}}{d_{R}-d_{L}} .
\end{array}
$$

Thus, for $T_{+}^{\prime}\left(d_{L}\right)>-1$ (resp. $\left.T_{+}^{\prime}\left(d_{L}\right)<-1\right)$ the fixed point $x_{M}^{*}$ appears attracting (resp. repelling).

Similarly, in case (ii) where $W\left(d_{R}\right)=B\left(d_{R}\right)$ and the border collision condition $d_{R}\left(a-a_{R}\right)=-a_{R}$ holds, the left derivative of function $T(x)$ in $d_{R}$ is given by

$$
\begin{array}{r}
T_{-}^{\prime}\left(d_{R}\right)=1+d_{R}\left(1-d_{R}\right) \frac{a-a_{M}}{a d_{R}+\delta}, \quad \text { where } \\
a_{M}=\frac{a d_{R}-\mu_{L}-a_{L} d_{L}}{d_{R}-d_{L}}
\end{array}
$$

and for $T_{-}^{\prime}\left(d_{R}\right)>-1\left(\operatorname{resp} . T_{-}^{\prime}\left(d_{R}\right)<-1\right)$ the fixed point $x_{M}^{*}$ appears attracting (resp. repelling).

As specified in Theorem 2, the only equilibrium of integration that may be locally attracting is the fixed point in the middle branch, $x_{M}^{*}$. Despite its existence and stability, the real possibility to have integration depends on the amplitude of its basin of attraction and on the amplitude of its existence/stability region in the parameter space (that we shall consider below). In fact, coexisting with at lest one stable equilibrium of segregation, the amplitude of the basin of attraction of the equilibrium of integration measures the real possibilities to observe patterns of residential integration. Then, comparing the four scenarios listed in Theorem 2, we conclude that the chances of residential integration are greater in the case of Scenario 3 where, considering for example the situation in Figure 2(first row), the basin of attraction of $x_{M}^{*}$, i.e. $\mathcal{B}\left(x_{M}^{*}\right)=\left(x_{L}^{*}, 1\right)$ with $x_{L}^{*}<0.5$, covers more than half of the state space $[0,1]$.

The basin $\mathcal{B}\left(x_{M}^{*}\right)$ of $x_{M}^{*}$ (assuming it is attracting) is reduced in the case of Scenario 4 , since at most it is given by the interval $\mathcal{B}\left(x_{M}^{*}\right)=\left(x_{L}^{*}, x_{R}^{*}\right)$. Considering for example the situation in Figure $3(\mathrm{~b})$, the equilibrium of integration $x_{M}^{*}$ is stable but its basin of attraction, $\mathcal{B}\left(x_{M}^{*}\right)=\left(x_{L}^{*}, x_{R}^{*}\right)$, is smaller than half of the state space $[0,1]$.

Let us further point out that in Scenarios 3 and 4 the possibilities of integration may decrease because disconnected basins of attraction appear through global bifur- cation which may occur when a critical point (image of finite rank of a kink point) merges with a fixed point, also called contact bifurcation. This kind of bifurcation changes the structure of the involved basins of attraction. For example, considering the case shown in Figure 2 (second row), we can see the basin $\mathcal{B}\left(x_{0}^{*}\right)=\left[0, x_{L}^{*}\right)$ while $\left[x_{L}^{*}, x_{R}^{*}\right]$ is an invariant interval (since $T\left(d_{L}\right)<x_{R}^{*}$ and $\left.T\left(d_{R}\right)>x_{L}^{*}\right)$ with non segregated dynamics inside. If the parameters are changed so to get $T\left(d_{R}\right)=x_{L}^{*}$ (contact bifurcation) after which $T\left(d_{R}\right)<x_{L}^{*}$, then the interval $\left[x_{L}^{*}, x_{R}^{*}\right]$ is no longer invariant and $\mathcal{B}\left(x_{0}^{*}\right)$ expands including also infinitely many intervals inside $\left(x_{L}^{*}, x_{R}^{*}\right)$. One more example is shown in Figure 3. In Figure $3(\mathrm{~b})$ we can see the basins $\mathcal{B}\left(x_{0}^{*}\right)=\left[0, x_{L}^{*}\right)$, $\mathcal{B}\left(x_{M}^{*}\right)=\left(x_{L}^{*}, x_{R}^{*}\right)$, which is an invariant interval (since $\left.T\left(d_{L}\right)<x_{R}^{*}\right)$, and $\mathcal{B}\left(x_{1}^{*}\right)=\left(x_{R}^{*}, 1\right]$. In Figure $3(\mathrm{c})$, as a consequence of the contact bifurcation occurring for $T\left(d_{L}\right)=x_{R}^{*}$, we have $T\left(d_{L}\right)>x_{R}^{*}$ and the basin $\mathcal{B}\left(x_{1}^{*}\right)$ expands, including a sequence of infinitely many intervals in $\left[x_{L}^{*}, x_{R}^{*}\right]$ accumulating to $x_{L}^{*}$, so that the set of initial conditions ultimately leading to segregation increases.

The above examples refer to cases of Scenario 4, for which there are three internal fixed points of non segregation, $x_{L}^{*}$ (repelling), $x_{M}^{*}$ and $x_{R}^{*}$ (repelling), and both contact bifurcations (with the local maximum $T\left(d_{L}\right)$ and the local minimum $T\left(d_{R}\right)$ ) can occur, also one after the other, expanding both the basins of segregation $\left(\mathcal{B}\left(x_{0}^{*}\right)\right.$ and $\left.\mathcal{B}\left(x_{1}^{*}\right)\right)$ and therefore reducing the possibility of integrated dynamics. Differently, in the case of Scenario 3 when there are two internal fixed points of non segregation, $x_{L}^{*}$ (repelling) and $x_{M}^{*}$, see an example in Figure 2(first row), we have only two basins of attraction: $\mathcal{B}\left(x_{0}^{*}\right)=\left[0, x_{L}^{*}\right)$ and the basin of bounded dynamics $\left(x_{L}^{*}, 1\right)$, which includes all the points having non segregated behavior. In fact, the limit set of the trajectories in $\left(x_{L}^{*}, 1\right)$ can include cycles, that is periodic orbits, as well as aperiodic ones, but always inside the interval $\left(x_{L}^{*}, T\left(d_{L}\right)\right)$ (since necessarily $\left.T\left(d_{L}\right)>x_{M}^{*}\right)$. Thus, the transition to disconnected basin may occur only via the transition $T\left(d_{R}\right) \gtrless x_{L}^{*}$.

The following Theorem indicates the conditions related to the occurrence of the contact bifurcations $T\left(d_{L}\right)=x_{R}^{*}$ and $T\left(d_{R}\right)=x_{L}^{*}$ associated with disconnected basins of attraction.

Theorem 3 Consider map $T$ as defined in (6) and the parameter conditions (2), (3) and (5), then:

1. In the case of Scenario 3 in Theorem 2 the basins of attraction are disconnected if $T\left(d_{R}\right)<x_{L}^{*}$. Moreover, $T\left(d_{R}\right)=x_{L}^{*}$ occurs for $d_{R}=d_{C B-R}$, where

$$
d_{C B-R}=\frac{\delta\left(a_{L}-a\right)+2 \mu_{L} a_{R}+\sqrt{\left(\delta\left(a-a_{L}\right)-2 \mu_{L} a_{R}\right)^{2}+4 \mu_{L}\left(\left(a-a_{L}\right) a+\mu_{L}\left(a_{R}-a\right)\right)\left(\delta-a_{R}\right)}}{2\left(\left(a-a_{L}\right) a+\mu_{L}\left(a_{R}-a\right)\right)} ;
$$



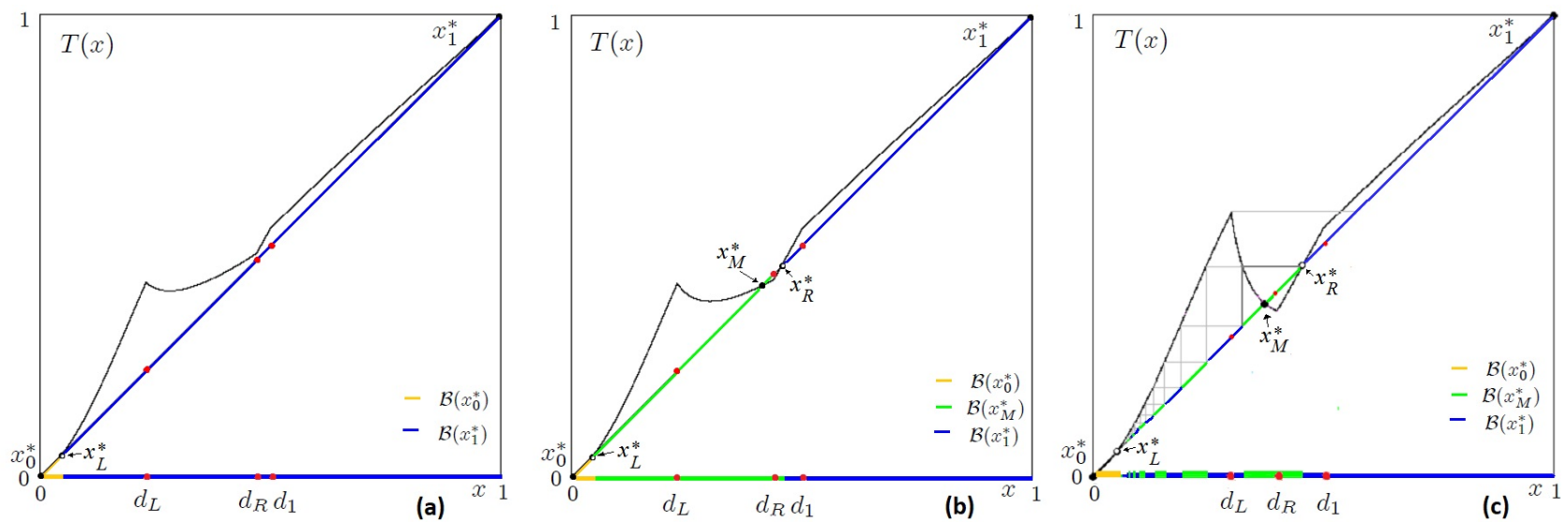

FIG. 3. Graph of map $T$ at $a=0.9, a_{L}=-0.1, a_{R}=-0.75, \mu_{L}=0.05, d_{L}=0.3$ with $d_{R}=0.46$ in Panel (a), $d_{R}=0.44$ in Panel (b) and $d_{R}=0.4$ in Panel (c). In all panels, black dots are the locally attracting fixed points, empty dots are the repelling ones and red dots represent the kink points $d_{L}, d_{R}$ and $d_{1}$. Moreover, the green region is the basin of attraction of the equilibrium of integration $x_{M}^{*}$, the blue region is the basin of attraction of the equilibrium of segregation $x_{1}^{*}$ and the orange interval is the basin of attraction of the equilibrium of segregation $x_{0}^{*}$.

two inequalities $T\left(d_{R}\right)<x_{L}^{*}$ and $T\left(d_{L}\right)>x_{R}^{*}$ holds.
$T\left(d_{R}\right)=x_{L}^{*}$ occurs for $d_{R}=d_{C B-R}$ given in $(17)$ while $T\left(d_{L}\right)=x_{R}^{*}$ occurs for $d_{L}=d_{C B-L}$ where

$$
d_{C B-L}=\frac{\delta\left(a_{R}-a\right)-a_{R}\left(a_{L}-\mu_{L}\right)+\sqrt{\left(\delta\left(a-a_{R}\right)-a_{R}\left(\mu_{L}-a_{L}\right)\right)^{2}-4 a_{R}\left(a^{2}-a_{R} a_{L}\right)\left(\mu_{L}+\delta\right)}}{2\left(a^{2}-a_{R} a_{L}\right)} .
$$

Theorem 3 underlines that the structure of the basins of attraction of map $T$ may be not so simple as reported in Figure 2(second row). In the example shown in Figure $3(\mathrm{c})$, where there are three internal fixed points of non segregation, $x_{M}^{*}$ is attracting, but $T\left(d_{L}\right)>x_{R}^{*}$ and thus the basins $\mathcal{B}\left(x_{M}^{*}\right)$ and $\mathcal{B}\left(x_{1}^{*}\right)$ are no longer connected. They are given by infinitely many disjoint intervals accumulating to $x_{L}^{*}$. The immediate basin of $x_{M}^{*}$ is now bounded by $x_{R}^{*}$ and its rank-1 preimage $x_{R}^{*,-1}$, i.e. is given by the interval $\left(x_{R}^{*,-1}, x_{R}^{*}\right)$, and the total basin $\mathcal{B}\left(x_{M}^{*}\right)$ consists in this interval and all its preimages of any rank. Similarly, the basin $\mathcal{B}\left(x_{1}^{*}\right)$ includes the interval $\left(x_{R}^{*}, 1\right]$ as well as infinitely many disjoint intervals, preimages of $\left(x_{R}^{*}, T\left(d_{L}\right)\right]$ accumulating to $x_{L}^{*}$.

Due to the disconnected basins of attraction the possibility of residential segregation increases and the possibility of integration can even reduce to zero. In the cases of Scenario 4, bounded integrated dynamics certainly exist when the interval $\left[T\left(d_{R}\right), T\left(d_{L}\right)\right]$ is invariant (and thus $\mathcal{B}\left(x_{0}^{*}\right)=\left[0, x_{L}^{*}\right)$ while $\left.\mathcal{B}\left(x_{1}^{*}\right)=\left(x_{R}^{*}, 1\right]\right)$ ). Clearly, the fixed point of non segregation $x_{M}^{*}$ may lose stability through a smooth flip bifurcation, and different attracting sets may appear inside the absorbing interval $\left[T\left(d_{R}\right), T\left(d_{L}\right)\right]$ in which the restriction of the map has a bimodal shape. As a function of the parameters, one of the two contact bifurcations indicated in Theorem 3 may generate disconnected basins of attraction, but still with some bounded attracting sets of integrated dynamics. When also the second one occurs (i.e. when both the two inequalities $T\left(d_{R}\right)<x_{L}^{*}$ and $T\left(d_{L}\right)>x_{R}^{*}$ hold), then the second contact has the effect of a final bifurcation since inside the interval $\left[T\left(d_{R}\right), T\left(d_{L}\right)\right]$ (no longer absorbing) only unstable sets are left, i.e. periodic or aperiodic repellors. Thus the invariant set of integration has a stable set of zero measure.

As in the classical Schelling's model, disconnected basins of attraction occur when the heterogeneity in the distributions of preferences for integration of the two groups of people increases and this reduces the possibility to have a pattern of integration.

The sensitivity of the model to parameter configurations is another fundamental aspect that influences integration. The amplitude of the stability region for the equilibrium of residential integration in the parameter space of the model is a second index of the robustness of dynamic patterns of residential integration. Bifurcation diagrams are a useful tool to study this aspect, both one-dimensional, showing the state $x_{t}$ as one parameter is varied, and two-dimensional, showing the bifurcation structure of the model as a function of two relevant parameters.

So, provided a short overview of the bifurcations that 
occur in piecewise smooth systems, we study the dynamics of our model when the equilibrium of segregation $x_{M}^{*}$ loses stability, which is essential to have a glimpse into the segregation patterns and to understand the robustness of the solution of integration. To investigate this aspect, let us consider the example of Scenario 4 in Figure 3(b) in which $x_{M}^{*}$ is stable and there are three internal fixed points and let us vary the two kink points $d_{L}$ and $d_{R}$. The two-dimensional bifurcation diagram so obtained is shown in Figure 4, where clearly only the region below the main diagonal is to be considered, i.e. the region $\left\{\left(d_{R}, d_{L}\right) \mid 0.5 \geq d_{R}>d_{L} \geq 0.1\right\}$. In this region of the parameter space we can study the transition from Scenario 4 to Scenario 2, or the vice versa, and the bifurcations that occur in these two scenarios, while Scenarios 1 and 3 cannot occur for these values of the parameters.

In Figure 4 the yellow color represents points of the vector $\left(d_{R}, d_{L}\right)$ of parameters related to convergence to the fixed point $x_{1}^{*}$ while the blue color represents convergence to the fixed point $x_{0}^{*}$ which we know are always coexisting attractors for the values of the parameters we are considering. For this constellation of the parameters, from (14) we have that the fold BCB occurs with the kink point $d_{R}$ at the value $d_{F B-R}=0 . \overline{45}$ (see the vertical line marked as $F-B C B_{R}$ in Figure 4). Decreasing $d_{R}$ and crossing this line we have the appearance of two internal fixed points $x_{R}^{*}$ and $x_{M}^{*}$. As we know, the first one is always repelling while $x_{M}^{*}$ may be either attracting or repelling, depending on the value of the left derivative in (16). We shall come back to the cases related to both repelling fixed points below, let us first analyze the case related to a stable fixed point of integration.

For parameter points in the green colored region of Figure 4 the fixed point $x_{M}^{*}$ is attracting and it coexists with the two stable equilibria of segregation $x_{0}^{*}$ and $x_{1}^{*}$ (as in the situation shown in Figure 3(b)). The basins of attraction of these three equilibria may be simply connected or not. As specified in Theorem 3, to have simply connected basins the map must be invariant in the interval $\left[x_{L}^{*}, x_{R}^{*}\right]$, i.e. $T\left(\left[x_{L}^{*}, x_{R}^{*}\right]\right) \subseteq\left[x_{L}^{*}, x_{R}^{*}\right]$, which implies $d_{L}<d_{C B-L}$ and $d_{R}<d_{C B-R}$. Thus, coming back to our example, in the green region below the line $d_{L}=d_{C B-L}$ (see the line $T\left(d_{L}\right)=x_{R}^{*}$ in Figure 4) the basins are connected, and given by $\mathcal{B}\left(x_{M}^{*}\right)=\left(x_{L}^{*}, x_{R}^{*}\right), \mathcal{B}\left(x_{0}^{*}\right)=\left[0, x_{L}^{*}\right)$ and $\mathcal{B}\left(x_{1}^{*}\right)=\left(x_{R}^{*}, 1\right]$ while for $d_{L}>d_{C B-L}$ in the green region the basins are given as in the example shown in Figure $3(\mathrm{c})$, i.e. $\mathcal{B}\left(x_{0}^{*}\right)=\left(0, x_{L}^{*}\right)$ while $\mathcal{B}\left(x_{M}^{*}\right)$ and $\mathcal{B}\left(x_{1}^{*}\right)$ consist of infinitely many disjoint intervals, which accumulate to $x_{L}^{*}$.

Starting in the green region and crossing its upper boundary (see the curve "s-flip $x_{M}^{*}$ " in Figure 4) the fixed point $x_{M}^{*}$ becomes unstable via a smooth flip bifurcation. Numerical investigations indicate that the bifurcation is a supercritical flip, leading to an attracting cycle of period 2, with periodic points in the middle branch, say $M M$ cycle, whose border collision with a kink point is indicated by the line "BC 2-cycle" in Figure 4, and will be commented below.

To better illustrate the occurring bifurcations, let us consider some one-dimensional bifurcation diagrams as functions of $d_{L}$. The one reported in Figure 5 illustrates the asymptotic dynamics of the model when $d_{L}$ varies along the red line in Figure 4 , at $d_{R}=0.25$ fixed. The ones in Figure 6 show the asymptotic dynamics of the model when $d_{L}$ varies along the blue line in Figure 4 , at $d_{R}=0.3$ fixed, and along the dark green line in Figure 4, at $d_{R}=0.35$ fixed.

These bifurcation diagrams underline that increasing the parameter $d_{L}$ the fixed point $x_{M}^{*}$ becomes unstable via a supercritical smooth flip bifurcation, as stated above, leading to an attracting 2-cycle with symbolic sequence $M M$, which in turn undergoes a border collision due to the contact of the smallest periodic point with the kink point $d_{L}$. The effect of this border collision depends on the two slopes of the second iterate $T^{2}(x)$ at the kink point $d_{L}$, and can be deduced by using the skew-tent map as a normal form. From Figure 4 we can see that when the pink color persists, then the collision leads to a persistent attracting 2-cycle with symbolic sequence $L M$, as shown in Figure 5, otherwise the resulting attracting set is different. In the two cases shown in Figure 6 the result of the $\mathrm{BCB}$ of the 2-cycle is transition to 2-cyclic chaotic intervals.

The bifurcation sequence observed in Figure 5 looks similar to those observable in the skew-tent map, but there are important differences due to the fact that here the branches are smooth and not linear. The 2-cycle that originates by supercritical flip bifurcation of $x_{M}^{*}$ undergoes (increasing $d_{L}$ ) a persistent $\mathrm{BC}$ (see also in Figure $7(\mathrm{a})$ ), and (further increasing $d_{L}$ ) a new pair of 2cycles having symbolic sequence $L R$ and $M R$ appear via fold $\mathrm{BCB}$, both repelling, as shown in Figure 7(b). As $d_{L}$ increases, the stable 2-cycle undergoes a second smooth flip bifurcation through which it loses stability and an attracting 4-cycle with symbolic sequence $L M L M$ appears, see Figure 7(c), and this is something which cannot occur in a piecewise linear map.

The attracting 4-cycle undergoes a BCB with the largest periodic point merging with $d_{R}$ and this bifurcation (shown in Figure 8(a)) leads to a chaotic attractor, i.e. 4-cyclic chaotic intervals. In Figure 8(b) 4 chaotic pieces can be seen which reduce to 2 chaotic pieces via a merging bifurcation (see Sushko, Gardini, and Avrutin (2016)) occurring at the homoclinic bifurcation of the unstable 2-cycle $L M$, as we can see in the enlargement of Figure 5, and in Figure 8(c).

The 2-cyclic chaotic intervals persist up to an expansion bifurcation (see Avrutin et al. (2014); Sushko, Gardini, and Avrutin (2016)) which occurs when two critical points on the boundary of the chaotic intervals merge with the repelling 2 -cycle with symbolic sequence $L R$, as shown in the enlargement of Figure 5, and in Figure 9(a), involving all the three branches below the kink point $d_{1}$, leading to a one-piece chaotic interval. The one-piece chaotic interval persists up to the final bifurcation of the 


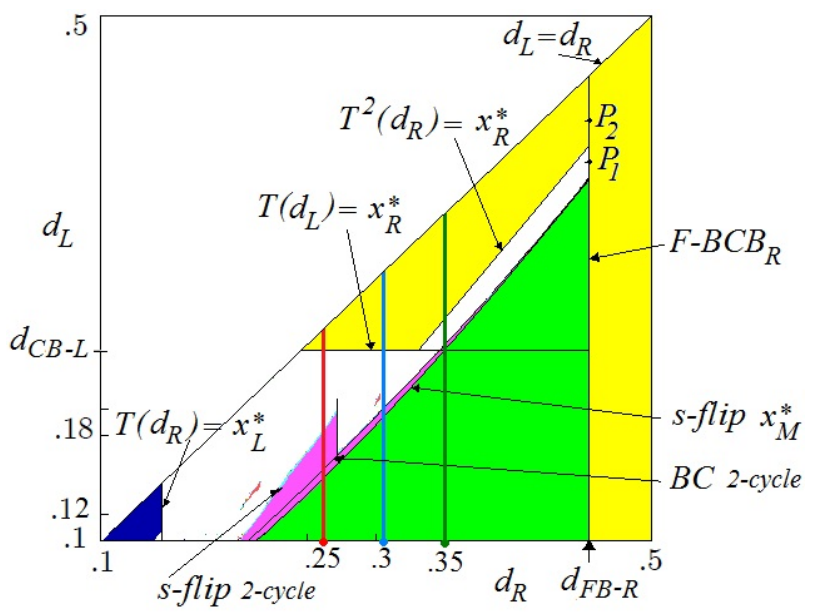

FIG. 4. Two-dimensional bifurcation diagram in the parameter plane $\left\{\left(d_{R}, d_{L}\right) \mid 0.5 \geq d_{R}>d_{L} \geq 0.1\right\}$ at $a=0.9, a_{L}=-0.1$, $\mu_{L}=0.05, a_{R}=-0.75$ fixed. In the blue (resp. yellow) the region in which the initial condition here used converges to $x_{0}^{*}$ (resp. $x_{1}^{*}$ ) (recall that the fixed points $x_{0}^{*}$ and $x_{1}^{*}$ are locally stable). In the green region $x_{M}^{*}$ is locally stable. The bifurcation diagram has been obtained taking an initial condition close to the fixed point $x_{M}^{*}$ when existing (i.e. for $\left.d_{R}<d_{F B-R}=0 . \overline{45}\right)$.

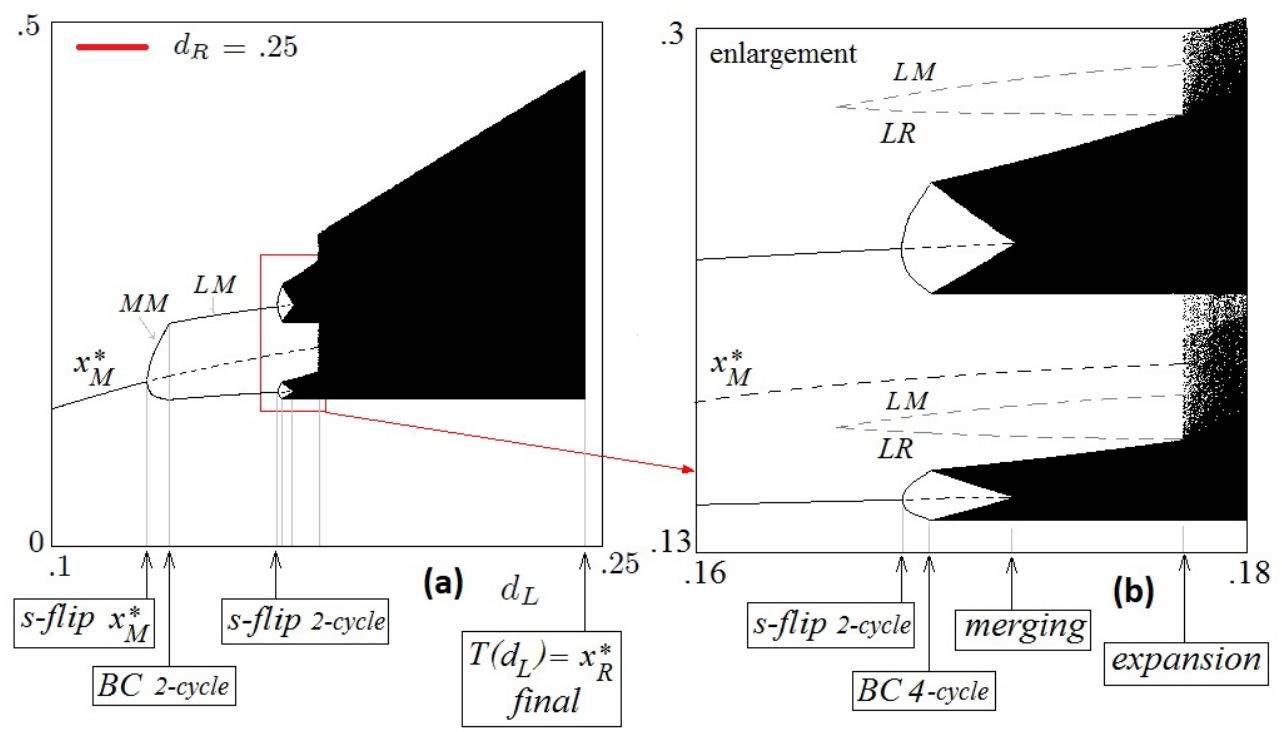

FIG. 5. One-dimensional bifurcation diagram along the red path in Figure 4 , at $d_{R}=0.25$ and $d_{L} \in(0.1,0.25)$, in Panel (a), with enlargement, in Panel (b). The other parameters as in Figure 4.

chaotic attractor, see Figure 5(a) and Figure 9(b), which here occurs at the contact bifurcation $T\left(d_{L}\right)=x_{R}^{*}$. This final bifurcation breaks the invariance of $T$ in the interval $\left[x_{L}^{*}, x_{R}^{*}\right]$, see Theorem 3 , whose effect is here to destroy the attracting set in the middle branch, leading to a chaotic repellor (see Figure 9(c)). Thus, for larger values of $d_{L}$ an invariant set $\Lambda$ with chaotic dynamics exists in $\left[x_{L}^{*}, x_{R}^{*}\right]$ and the two attracting sets are the equilibria of segregation, with basins given by $\mathcal{B}\left(x_{0}^{*}\right)=\left(0, x_{L}^{*}\right)$ and $\mathcal{B}\left(x_{1}^{*}\right)=\left(x_{L}^{*}, 1\right] \backslash \Lambda$ which is thus characterized by a fractal boundary.

The sequence of bifurcations through which the dynamics of integration change, underlines that integration is quite an unstable outcome that is very sensitive to the shapes of the distributions of preferences. Especially the final bifurcation, through which the attracting pattern of integration disappears, shows that distributions of preferences for which three equilibria of integration exist, namely Scenario 4 in Theorem 2, do not ensure the possibility of an integrated neighborhood. Thus, a social planner that wants to maximize integration has to be aware that people have to be educated to integration without exacerbating people's preferences for racially mixed neighborhoods. In fact, extreme feelings are responsible for overshooting dynamics, as disconnected basins of attraction, or final bifurcations, that increase the risk of segregation.

To avoid segregation it is important to detect the ex- 

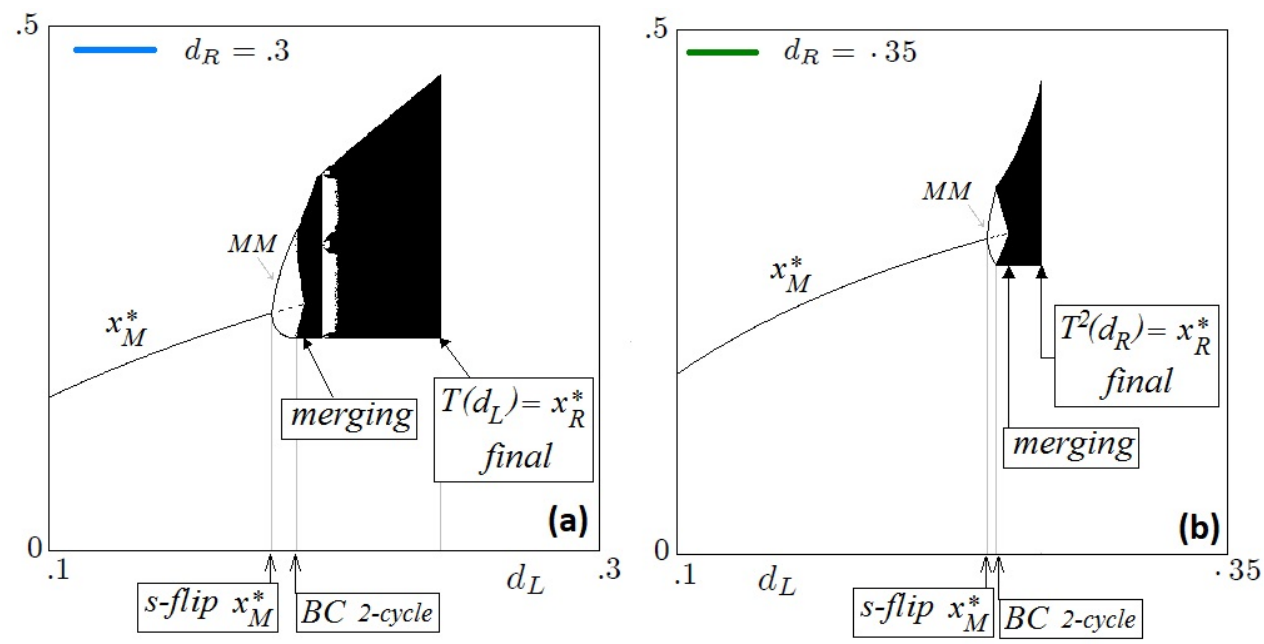

FIG. 6. One-dimensional bifurcation diagrams. In Panel (a) along the blue path in Figure 4 , at $d_{R}=0.3$ and $d_{L} \in(0.1,0.3)$. In Panel (b) along the dark green path in Figure 4 , at $d_{R}=0.35$ and $d_{L} \in(0.1,0.35)$. The other parameters as in Figure 4 .

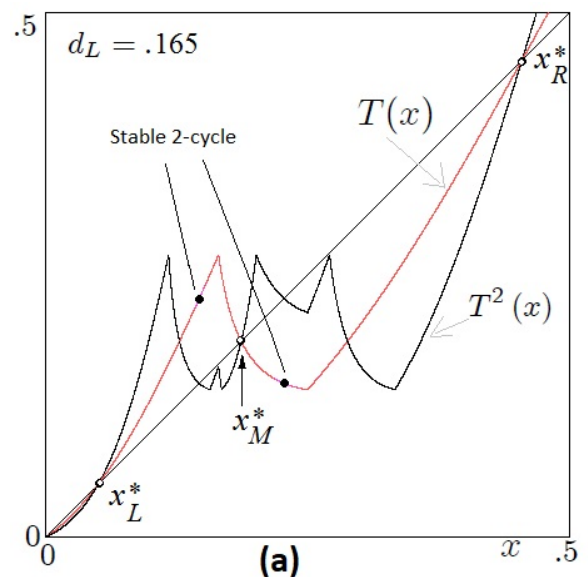

(a)

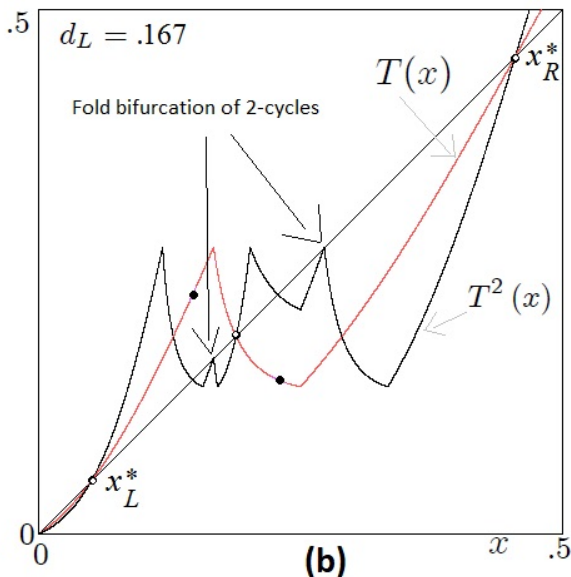

(b)

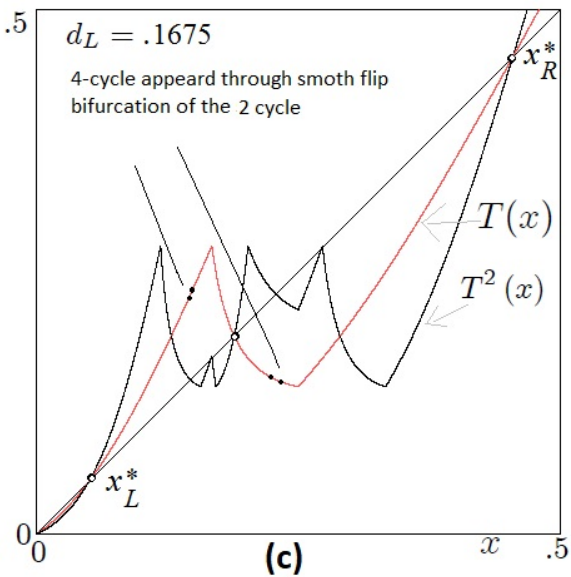

(c)

FIG. 7. Graphs of map $T$ in red and in black its second iterate $T^{2}$. Parameters as in Figure 5 and $d_{L}=0.165$ in Panel (a): stable 2-cycle originated by smooth flip bifurcation of $x_{M}^{*} ; d_{L}=0.167$ in Panel (b): example of fold BCB through which a pair of 2-cycles appears, here both unstable; $d_{L}=0.1675$ in Panel (c): a stable 4-cycle originated via smooth flip bifurcation of the 2-cycle depicted in Panel (a).

act value at which a final bifurcation occurs (i.e. the bifurcation leading only to segregation as attractors) and it is not always coinciding with the contact bifurcation $T\left(d_{L}\right)=x_{R}^{*}$. For instance, the final bifurcation of the chaotic attractor in the example shown in Figure $6(\mathrm{~b})$ is of a different type with respect to the one illustrated in Figure 6(a) and it occurs for larger values of $d_{R}$. In fact, it occurs after the contact bifurcation $T\left(d_{L}\right)=x_{R}^{*}$ which breaks the invariance of $T$ in the interval $\left[x_{L}^{*}, x_{R}^{*}\right]$ (and whose effect is to modify the structure of the basins of the two attractors different from $\left.x_{0}^{*}\right)$. In Figure 10(a) we can see that $\left(x_{R}^{*,-1}, x_{R}^{*}\right)$ is the immediate basin of the one-piece chaotic attractor $\left[T\left(d_{R}\right), T^{2}\left(d_{R}\right)\right]$, and the final bifurcation occurs at the contact $T^{2}\left(d_{R}\right)=x_{R}^{*}$, here also homoclinic bifurcation of $x_{R}^{*}$, shown in Figure 10(b), where the chaotic interval is $\left[T\left(d_{R}\right), T^{2}\left(d_{R}\right)\right]=\left[x_{R}^{*,-1}, x_{R}^{*}\right]$. After the bifurcation, a chaotic repellor $\Lambda$ of zero Lebesgue measure remains in the interval $\left[x_{R}^{*,-1}, x_{R}^{*}\right]$.

This second type of final bifurcation explains also the difference between the white and the yellow region when crossing the vertical line $d_{R}=d_{F B-R}(=0 . \overline{45})$ in Figure 4 , which is the fold $\mathrm{BCB}$ through which two more internal fixed points $x_{M}^{*}$ and $x_{R}^{*}$ appear, and $x_{M}^{*}$ can be either attracting or repelling. As already remarked, crossing the vertical line $d_{R}=d_{F B-R}$ and entering the green region, the fixed point $x_{M}^{*}$ is attracting. Differently, crossing the same line entering in the white or yellow region (as in the points $P_{1}$ and $P_{2}$ of Figure 4) the two new fixed points that appear at the fold $\mathrm{BCB}$ are both repelling. The difference between the two transitions (to the white or yellow region) is in the appearance of a chaotic attractor or of a chaotic repellor, as shown in Figure 11. In Figure 11(a), where $T^{2}\left(d_{R}\right)<x_{R}^{*}$, a chaotic 

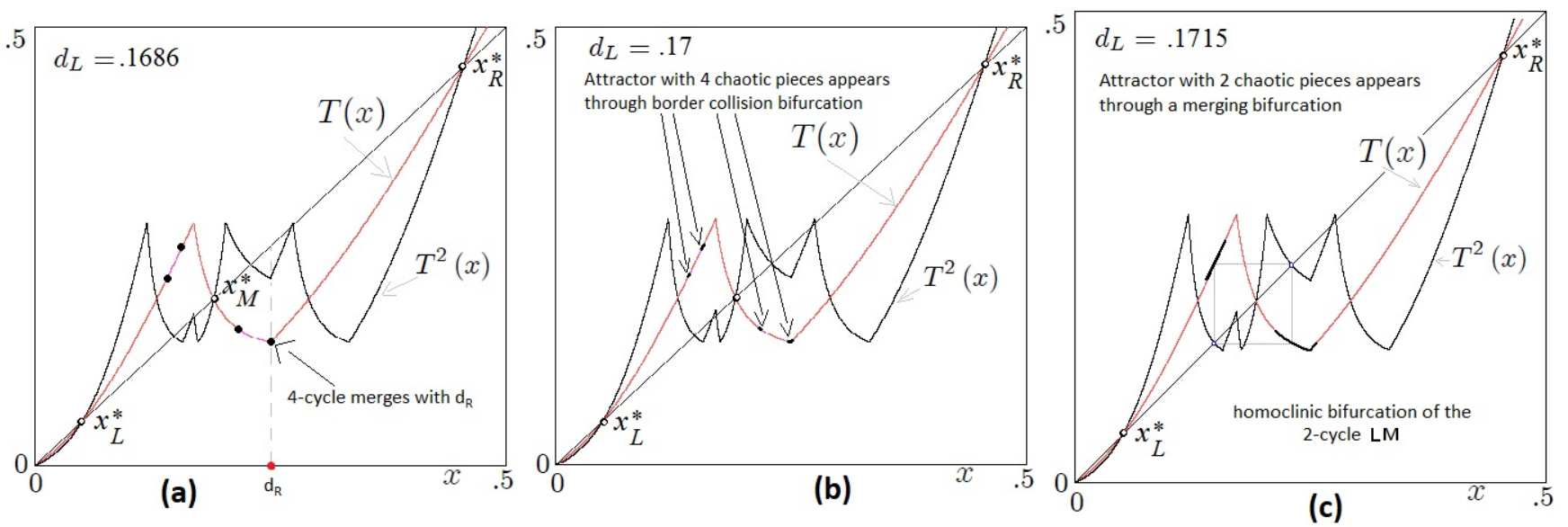

FIG. 8. Graphs of map $T$ in red and in black its second iterate $T^{2}$. Parameters as in Figure 5 and $d_{L}=0.1686$ in Panel (a): $\mathrm{BCB}$ of the stable 4-cycle, leading to 4-cyclic chaotic intervals shown in Panel (b) where $d_{L}=0.17 ; d_{L}=0.1715$ in Panel (c): merging bifurcation leading to two chaotic intervals, homoclinic bifurcation of the 2-cycle $L M$ at which the 4 pieces of the 4-cyclic chaotic intervals shown in Panel (b) merge.
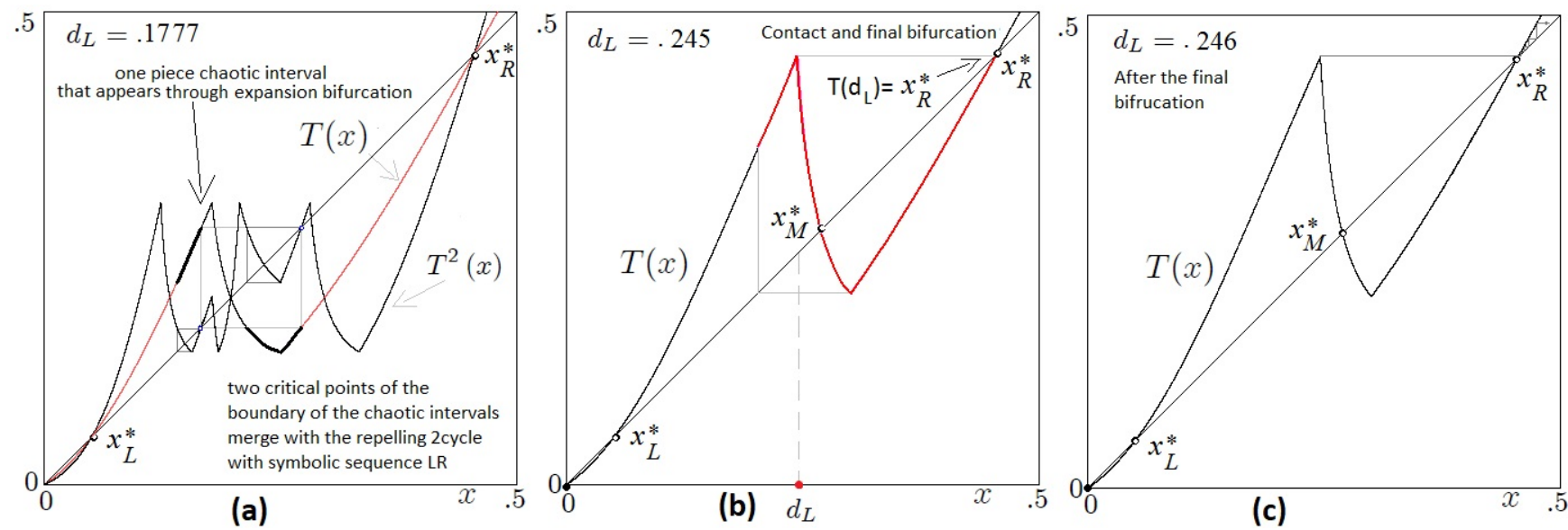

FIG. 9. Parameters as in Figure 5 and $d_{L}=0.1777$ in Panel (a): the attractor consists in one-piece chaotic interval appeared through an expansion bifurcation at $d_{L}=0.1776$, homoclinic bifurcation of the 2-cycle $L R$ after which the chaotic set expands to $\left[T\left(d_{R}\right), T\left(d_{L}\right)\right]$; Panel (b) at $d_{L}=0.245$ : final bifurcation at $T\left(d_{L}\right)=x_{R}^{*} ; d_{L}=0.246$ in Panel (c): after the final bifurcation almost all the trajectories in $\left(x_{L}^{*}, 1\right]$ are converging to the segregation equilibrium $x^{*}=1$ except for a chaotic repellor $\Lambda$ of zero measure.

interval $\left[T\left(d_{R}\right), T^{2}\left(d_{R}\right)\right]$ exists in $\left(x_{R}^{*,-1}, x_{R}^{*}\right)$ and it is attracting, while in Figure 11(b), where $T^{2}\left(d_{R}\right)>x_{R}^{*}$, a chaotic repellor exists in $\left(x_{R}^{*,-1}, x_{R}^{*}\right)$.

The second contact bifurcation indicated in Theorem 3 occurs at $d_{R}=d_{C B-R}$, when $T\left(d_{R}\right)=x_{L}^{*}$, and it is relevant at low values of $d_{R}$, as it is evident from Figure 4. The bifurcation diagram in Figure 12(a) shows this bifurcation. Here $d_{L}=0.12$ is fixed and $d_{R}$ varies in the interval $\left(d_{L}, d_{1}\right)$. For $d_{R}$ slightly larger than $d_{C B-R}$, a stable chaotic interval exists. This attracting chaotic interval appears/disappears through the contact bifurcation occurring at $d_{R}=d_{C B-R}=0.145$. Decreasing $d_{R}$ (starting with $d_{R}>d_{C B-R}$ ), the chaotic attractor becomes a chaotic repellor on the boundary of the equilibrium, so-called final bifurcation. Vice versa, increasing $d_{R}$, (starting with $d_{R}<d_{C B-R}$ ) a chaotic repellor be- comes a chaotic attractor. After the contact (here homoclinic bifurcation of $x_{L}^{*}$ ), for $d_{R}<0.145$, it is the basin of attraction of $x_{0}^{*}$ which becomes wider and with a fractal boundary. In fact, after the contact an invariant set $\Lambda$ with chaotic dynamics exists in $\left[x_{L}^{*}, x_{R}^{*}\right]$ and the two attracting sets are the two equilibria of segregation, with basins given by $\mathcal{B}\left(x_{0}^{*}\right)=\left(0, x_{R}^{*}\right) \backslash \Lambda$, which is thus characterized by a fractal boundary, and $\mathcal{B}\left(x_{1}^{*}\right)=\left(x_{R}^{*}, 1\right)$.

Let us conclude the bifurcation analysis of the model by underlining the complex interplay between smooth bifurcations and BCBs which can be appreciated from the case shown in Figure 12(b) with enlargement in Figure $12(\mathrm{c})$. Here, for $d_{L}=0.18$ fixed and varying $d_{R}$ in the interval $\left(d_{L}, d_{1}\right)$, it is shown that decreasing $d_{R}$ the 2-cycle $M M$ born at the smooth flip bifurcation of the fixed point $x_{M}^{*}$ also undergoes a cascade of smooth 

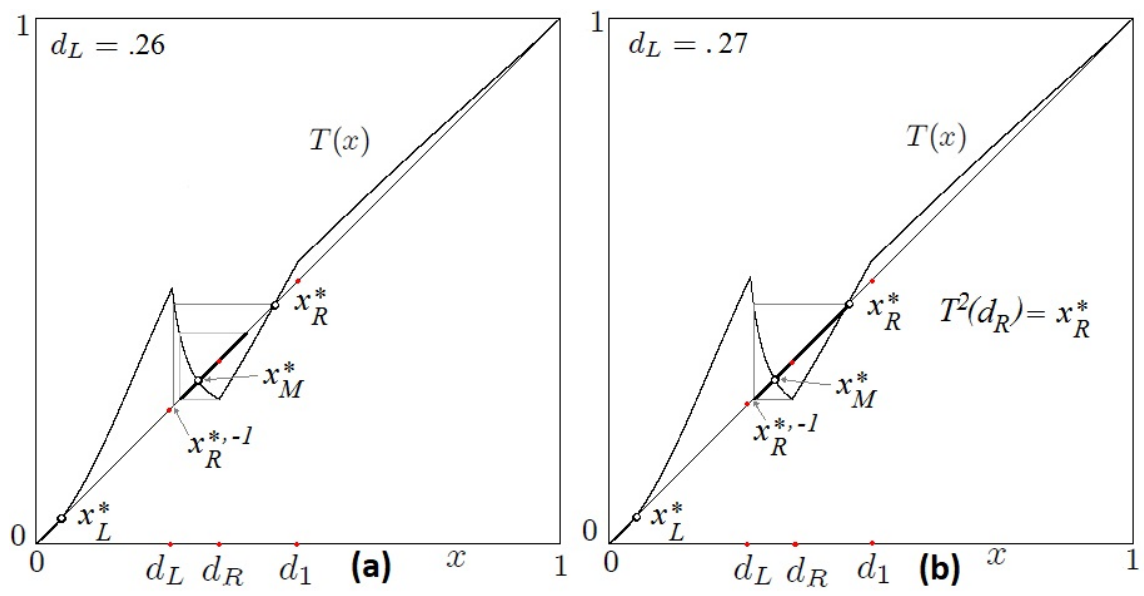

FIG. 10. Parameters as in Figure 6 and $d_{L}=0.26$ in Panel (a): disconnected basins of two attractors consisting in one-piece chaotic interval and $x^{*}=1$; Panel (b) at $d_{L}=0.27$ : after the final bifurcation occurring when $T^{2}\left(d_{R}\right)=x_{R}^{*}$, almost all the trajectories in $\left(x_{L}^{*}, 1\right]$ are converging to the segregation equilibrium $x^{*}=1$ except for a chaotic repellor $\Lambda$ of zero measure.
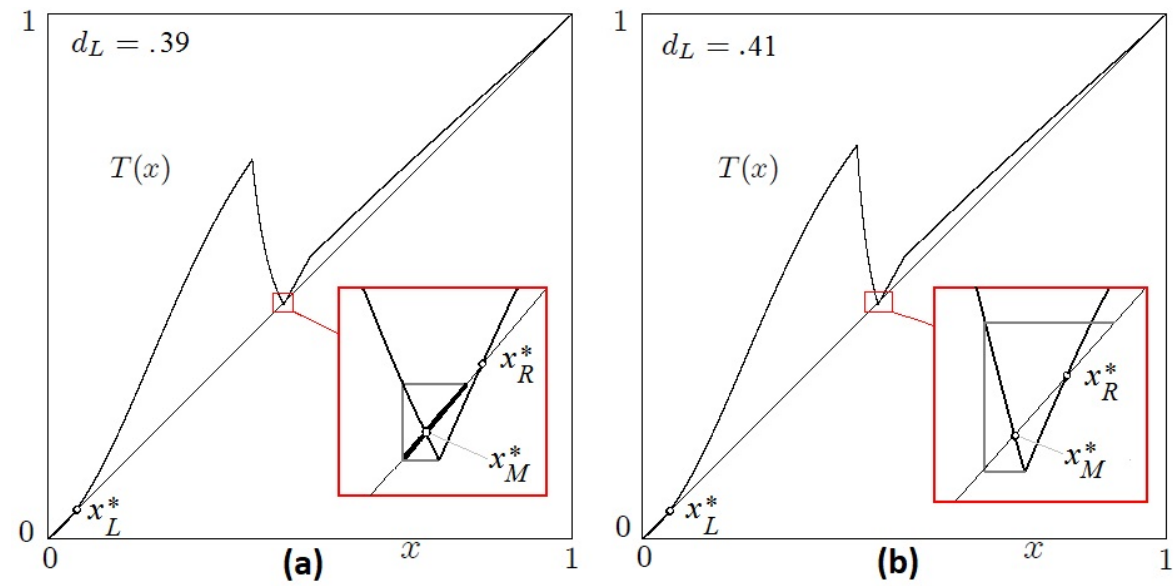

FIG. 11. Parameters as in Figure $4, d_{R}=d_{F B-R}=0 . \overline{45}$ and two values of the vector $\left(d_{R}, d_{L}\right)$ given by $P_{1}=\left(d_{F B-R}, 0.39\right)$ and $P_{2}=\left(d_{F B-R}, 0.41\right)$ which are indicated in the two-dimension bifurcation digram of Figure 4 . Panel (a), the result of the fold $\mathrm{BCB}$ in $P_{1}$ leading to a one-piece chaotic attractor. Panel (b), the result of the fold BCB in $P_{2}$ leading to a chaotic repellor.

bifurcations and BCBs. Worth mentioning is also the attracting 2-cycle $L M$ visible in Figure 12(b), which is here born differently from the cases shown above, that is, via a fold $\mathrm{BCB}$ of the second iterate $T^{2}$ at the kink point $d=d_{R}$ leading to a pair of 2-cycles, $L M$ attracting (visible in Figure 12(f)) and $L R$ repelling, and this is typical of a bimodal map. This repelling 2-cycle $L R$ is also responsible of the expansion bifurcation of the two-pieces chaotic attractor into a one-piece, as shown in the inset at $d_{R}=0.252$.

The conducted investigation of the local and global dynamics of the model reveals the different ways in which stable dynamics of integration appear and evolve. That is, fixed points of integration appear, stable or unstable, and different forms of patterns of integration may exist, showing the complicated dynamics related to an integrated neighborhood. These aspects have normative implications for a policy maker that wants to promote integration. In this respect, one aspect deserves particular attention and it is the shape of the distributions of preferences for integration. The bifurcation diagrams, reported and described in this section, show that the equilibrium of integration loses stability when $d_{L}$ and $d_{R}$ get close. This implies that the distribution of preferences of whites has a minimum point close to a maximum point, i.e. a sharp change in the degree of satisfaction for neighborhoods with similar compositions of the population. In this case the dynamics of the model can show a fast convergence to segregation. A route to segregation that is similar to the one of the threshold behavior of the Schelling's models. A more even distribution of preferences for integration of whites may avoid such macro pattern of segregation. A finding that is confirmed by empirical evidences, see, e.g., Bruch and Mare (2006). 

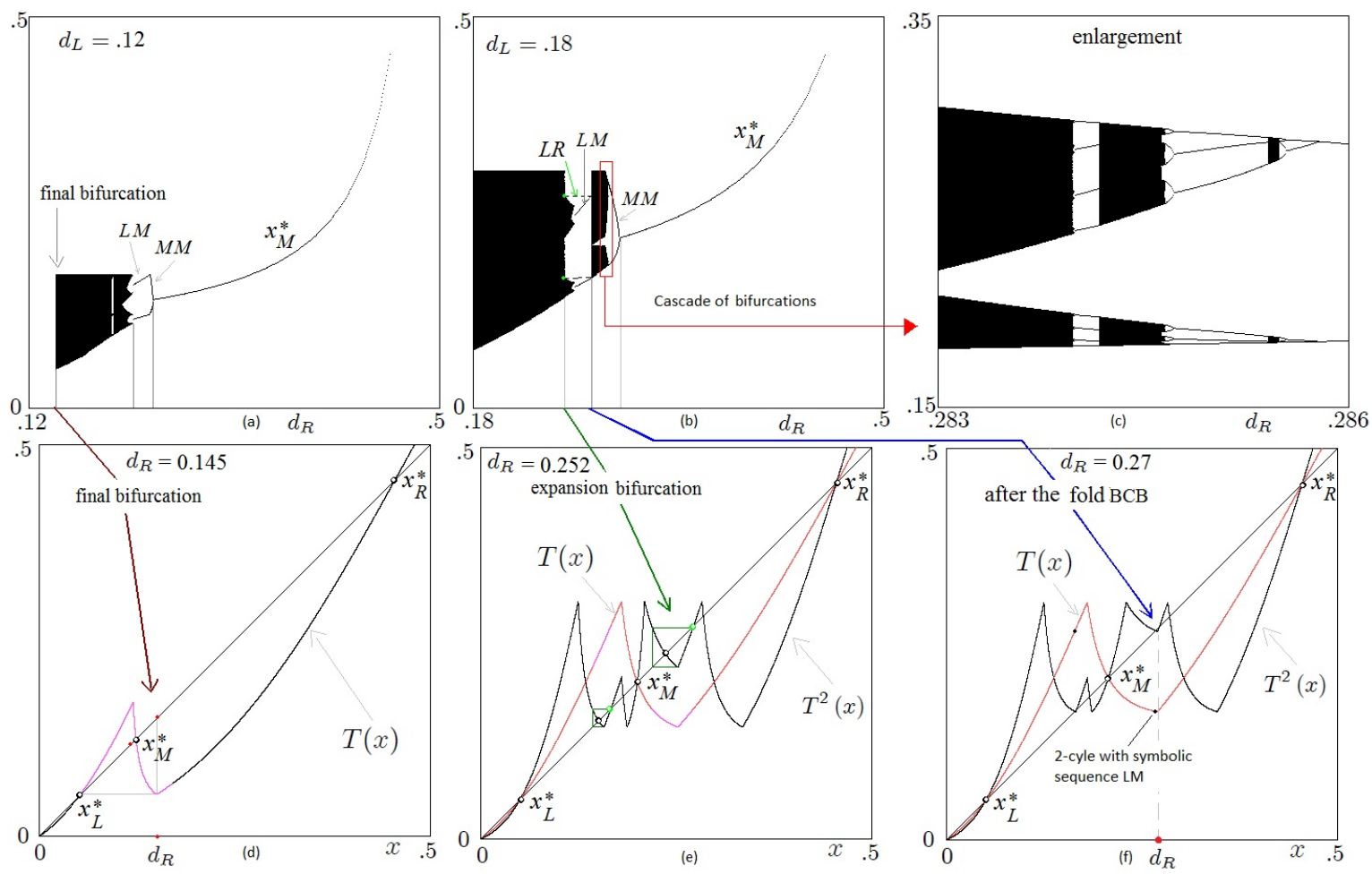

FIG. 12. Bifurcation diagrams varying $d_{R}$. Parameters as in Figure 4 and $d_{L}=0.12$ with $d_{R} \in(0.12,0.5)$ in Panel (a), $d_{L}=0.18$ with $d_{R} \in(0.18,0.5)$ in Panel (b), with an enlargement in Panel (c) for $d_{R} \in(0.283,0.286)$. Inset for Panel (a) at $d_{R}=0.145$ : final bifurcation. Insets for Panel (b) at $d_{R}=0.252$ : expansion bifurcation of the two-pieces chaotic attractor into a one-piece chaotic attractor, and at $d_{R}=0.27$ : soon after the fold BCB related to a pair of 2-cycles, one attracting and one repelling.

\section{CONCLUSIONS}

Residential segregation is observed in many metropolitan areas of the western society despite people express their preferences for integration. The dynamic models proposed by Schelling, see e.g. Schelling (1969, 1971), provide a theoretical explanation for the discrepancies between macro patterns of segregation and individual preferences for integration. Schelling's models are based on tolerances and on the threshold behavior according to which people change the residential location if the racial composition of the district crosses the threshold level of tolerance and enter otherwise.

In the current paper, we propose an evolutionary version of the Schelling's model where agents decide their residential location according to their individual distributions of preferences which represent their payoff functions. Agents' location decision is modeled by a replicator dynamics according to which agents chase the best relative performance. Despite the differences, our evolutionary game shows patterns of segregation that are similar to the segregation dynamics of the Schelling's models. In fact, it shows that segregation, despite being a suboptimal equilibrium of the game, is stable and a racially diverse neighborhood is possible only for peculiar configurations of the distributions of preferences and, even when it exists, it is highly sensitive to parameter variations and initial conditions. Then, our analysis provides an evolutionary justification to the segregation dynamics, showing that segregation can arise through the so-called spiteful behaviors, see, e.g., Massey and Denton (1987).

Compared to the Schelling's neighborhood-tipping model, which is a two-dimensional dynamical system, see, e.g., Bischi and Merlone (2011), the evolutionary game here proposed takes the form of a one-dimensional map. It is continuous and piecewise smooth, with two kink points which highly influence the observed dynamics.

We have performed the detailed analysis of the possible fixed points of non segregation, and the related stability, showing that only one equilibrium of integration can be stable. Moreover, when the equilibrium is repelling, interesting integrated dynamics may exist, and we have described the relevant bifurcations depending on two parameters (the two kink points of the map). By using the piecewise smooth character of the model some bifurcations can be explained, such as the BCBs related to the fixed points, which are global bifurcations or homoclinic bifurcations related to disconnected basins of attraction; the final bifurcation (since it leads to no attracting integrated dynamics); as well as some new bifurcation structures still to be investigated in detail, since the interplay between smooth bifurcations and border collision bifurca- 
tions is a new subject which deserve attention from the Scholars.

Let us finally point out that our one-dimensional model can be easily generalized by introducing for example a third group of agents, such as the Asian or the Hispanic population. This would be an interesting extension of the current model that can help to shed some lights on the reasons behind the high levels of segregation that concern the blacks and the small levels of segregation that involve the Hispanic or Asian population in the U.S. metropolitan areas, see, e.g., Massey and Denton (1987). Sociologists ascribe the higher level of integration of the Hispanics to their distribution of preferences for integration that differs from the one of the blacks and is similar to the one of the whites, see, e.g., Clark (1991). We leave for future works the description and analysis of a model with three groups of agents, such as blacks, whites and Hispanics, which allows to verify this conjecture at least at a theoretical level. At the same time, in future works the agents' decision mechanism can be modified to take into account the costs of changing residence, such as find a new flat to rent, modify habits, leave people and, eventually, this cost function can be described by an increasing function with respect to the number of movements. Alternatively, it would be interesting to consider that, after each movement, an agent revises her/his preference, for instance by becoming more tolerant instead of moving again.

\section{ACKNOWLEDGMENTS}

The authors thank Gian-Italo Bischi, Luca Colombo, Philipp Harting, Alan Kirman and all participants in the The GeComplexity Conference held at the University of Crete for their insightful comments and suggestions that have lead to several improvements in the paper. All remaining mistakes are our own. The work has been developed in the framework of the research project on Dynamic Models for Behavioral Economics financed by DESP-University of Urbino. The work of L. Gardini has been done within the activities of the GNFM (National Group of Mathematical Physics, INDAM Italian Research Group).

Avrutin, V., Gardini, L., Schanz, M., and Sushko, I., "Bifurcations of chaotic attractors in one-dimensional piecewise smooth maps," International Journal of Bifurcation and Chaos 24, 1440012 (10 pages) (2014).

Avrutin, V., Sushko, I., and Gardini, L., "Cyclicity of chaotic attractors in one-dimensional discontinuous maps," Mathematics and Computers in Simulation 95, 126-136 (2014).

Banerjee, S., Yorke, J. A., and Grebogi, C., "Robust chaos," Physical Review Letters 80, 3049-3052 (1998).

Bischi, G.-I. and Merlone, U., "Nonlinear economic dynamics," (Nova Science Publisher, 2011) Chap. An adaptive dynamic model of segregation, pp. 191-205.

Bossan, B., Jann, O., and Hammerstein, P., "The evolution of social learning and its economic consequences," Journal of Economic Behavior \& Organization 112, 266-288 (2015).
Bruch, E. E. and Mare, R. D., "Neighborhood choice and neighborhood change," American Journal of Sociology 112, 667-709 (2006).

Clark, W. A. V., "Residential preferences and neighborhood racial segregation: A test of the Schelling segregation model," Demography 28, 1-19 (1991).

Davis, J. A. and Smith, T. W., "General social surveys, 1972-1994," Tech. Rep. (Machine-readable data file, National Opinion Research Center, Chicago (producer), Storrs, C. T., The Roper Center for Public Opinion Research (distributor), 1994).

Dokumaci, E. and Sandholm, W. H., "Shelling redux: An evolutionary dynamic model of residential segregation," (2006), University of Wisconsin-Madison.

Epstein, J. M. and Axtell, R., Growing Artificial Societies: Social Science from the Bottom Up (Cambridge, MA: MIT Press, 1996).

Fagiolo, G., Valente, M., and Vriend, N. J., "Segregation in networks," Journal of Economic Behavior \& Organization 64, 316336 (2007).

Glaeser, E. L. and Vigdor, J. L., "Racial segregation in the 2000 Census: Promising news." Brookings Institution Survey Series. Washington: Brookings Institution (2001).

Hamilton, W. D., "Selfish and spiteful behavior in an evolutionary model," Nature 228, 1218-1220 (1970).

Harris, D., "'property values drop when blacks move in, because ...': Racial and socioeconomic determinants of neighborhood desirability," American Sociological Review 64, 461-479 (1999).

Hofbauer, J. and Sigmund, K., "Evolutionary game dynamics," Bulletin of the American Mathematical Society 40, 479-519 (2003).

Krysan, M. and Farley, R., "The residential preferences of blacks: Do they explain persistent segregation?" Social Forces 80, 937980 (2002).

Logan, J. R., Stults, B. J., and Farley, R., "Segregation of minorities in the metropolis: Two decades of change," Demography 41, 1-22 (2004).

Maistrenko, Y. L., Maistrenko, V. L., and Vikul, S. I., "On periodadding sequences of attracting cycles in piecewise linear maps," Chaos, Solitons \& Fractals 9, 67-75 (1998).

Massey, D. and Denton, N. A., American Apartheid: Segregation and the Making of the Underclass (Cambridge, MA: Harward University Press, 1993).

Massey, D. S. and Denton, N. A., "Trends in the residential segregation of Blacks, Hispanics, and Asians: 1970-1980," American Sociological Review 52, 802-825 (1987).

Nusse, H. E. and Yorke, J. A., "Border-collision bifurcations for piecewise smooth one-dimensional maps," International Journal of Bifurcation and Chaos 5, 189-207 (1995).

Panchuk, A., Sushko, I., Schenke, B., and Avrutin, V., "Bifurcation structures in a bimodal piecewise linear map: Regular dynamics," International Journal of Bifurcation and Chaos 23, 1330040 (26 pages) (2013).

Pancs, R. and Vriend, J. N., "Schelling's spatial proximity model of segregation revisited," Journal of Public Economics 2007, 1-24 (91).

Radi, D. and Gardini, L., "Entry limitations and heterogeneous tolerances in a Schelling-like segregation model," Chaos, Solitons \& Fractals 79, 30-144 (2015).

Radi, D., Gardini, L., and Avrutin, V., "The role of constraints in a segregation model: The asymmetric case," Discrete Dynamics in Nature and Society Article ID 569296, 17 pages (2014a).

Radi, D., Gardini, L., and Avrutin, V., "The role of constraints in a segregation model: The symmetric case," Chaos, Solitons \& Fractals 66, 103-119 (2014b).

Schaffer, M. E., "Are profit-maximisers the best survivors?: A Darwinian model of economic natural selection," Journal of Economic Behavior \& Organization 12, 29-45 (1989).

Schelling, T. C., "Models of segregation," The American Economic Review 59, 488-493 (1969).

Schelling, T. C., "Dynamic models of segregation," The Journal of 
Mathematical Sociology 1, 143-186 (1971).

Schelling, T. C., Micromotives and Macrobehavior (New York: W. W. Norton, 1978).

Schuman, H., Steeh, C., Bobo, L. D., and Krysan, M., Racial Attitudes in America: Trends and Interpretations. Revised ed. (Cambridge, MA: Harvard University Press, 1997).

Sushko, I., Avrutin, V., and Gardini, L., "Bifurcation structure in the skew tent map and its application as a border collision normal form," Journal of Difference Equations and Applications
22, 1040-1087 (2016).

Sushko, I., Gardini, L., and Avrutin, V., "Nonsmooth onedimensional maps: Some basic concepts and definitions," Journal of Difference Equations and Applications 22, 1816-1870 (2016).

Zhang, J., "Residential segregation in an all-integrationist world," Journal of Economic Behavior \& Organization 54, 533-550 (2004).

Zhang, J., "Tipping and residential segregation: A unified Schelling model," Journal of Regional Science 51, 167-193 (2011). 